A Unified Perspective on Resource Allocation:

Limited Arbitage is Necessary and Sufficient for the Existence of a Competitive Equilibrium, the Core and Social Choice

by

Graciela Chichilnisky, Columbia University

June 1994, revised July 1996

Discussion Paper Series No. 9596-28

$d p 9596-28$

proge: 51 


\title{
A Unified Perspective on Resource Allocation: Limited Arbitrage is Necessary and Sufficient for the Existence of a Competitive Equilibrium, the Core and Social Choice
}

Graciela Chichilnisky*

Columbia University and UNESCO

First version June 1994, revised July $1996^{+}$

\begin{abstract}
Different forms of resource allocation-by markets, cooperative games, and by social choice-are unified by one condition, limited arbitrage, which is defined on the endowments and the preferences of the traders of an Arrow Debreu economy. Limited arbitrage is necessary and sufficient for the existence of a competitive equilibrium in economies with or without short sales, and with finitely or infinitely many markets. The same condition is also necessary and sufficient for the existence of the core, for resolving Arrow's paradox on choices of large utility values, and for the existence of social choice rules which are continuous, anonymous and respect unanimity, thus providing a unified perspective on standard procedures for resource allocation. When limited arbitrage does not hold, social diversity of various degrees is defined by the properties of a topological invariant of the economy, the cohomology rings $\mathrm{CH}$ of a family of cones which are naturally associated with it. $C H$ has additional information about the resource allocation properties of subsets of traders in the economy and of the subeconomies which they span.
\end{abstract}


Social diversity is central to resource allocation. People trade because they are different. Gains from trade and the scope for mutually advantageous reallocation depend naturally on the diversity of the traders' preferences and endowments. The market owes its existence to the diversity of those who make up the economy.

An excess of diversity could however stretch the ability of economic institutions to operate efficiently. This is a concern in regions experiencing extensive and rapid migration, such as Canada, the USA and the ex-USSR. Are there natural limits on the degree of social diversity with which our institutions can cope? This paper will argue that there are. I will argue that not only is a certain amount of diversity essential for the functioning of markets, but, at the other extreme, that too much diversity of a society's preferences and endowments may hinder its ability to allocate resources efficiently.

Somewhat unexpectedly, the very same level of diversity which hinders the functioning of markets also hinders the functioning of democracy, and other forms of resource allocation which are obtained through cooperative games, such as the core. ${ }^{1}$ The main tenet of this paper is that there is a crucial level of social diversity which determines whether all these forms of resource allocation will function properly.

Social diversity has been an elusive concept until recently. I give here a precise definition, and examine its impact on the most frequently used forms of resource allocation. From this analysis a new unified perspective emerges: a well-defined connection between resource allocation by markets, games and social choices, which have been considered distinct until now. I define a limitation on social diversity which links all these forms of resource allocation. This limitation is a condition on the endowments and the preferences of the traders of an Arrow Debreu economy. In its simpler form I call this limited arbitrage ${ }^{2}$. This concept is related with that of "no-arbitrage" ${ }^{3}$ used in finance, but it is nonetheless different from it. I show that limited arbitrage is necessary and sufficient for the existence of an equilibrium in Arrow Debreu economies, and this equivalence extends to economies with or without short sales ${ }^{4}$ and with finitely or infinitely many markets, ${ }^{5}$ Theorems 2 and 5 . Limited arbitrage is also necessary and sufficient for the existence of the core, ${ }^{6}$ Theorem 7 , and its simplest failure is sufficient for the existence of the supercore, a concept which is introduced to gauge social cohesion, Theorem 8. In addition, limited arbitrage is necessary and sufficient for solving Arrow's paradox (Arrow, 1951) on choices of large utility value, i.e. for the existence of well-defined social choice rules, ${ }^{7}$ Theorem 9. It is also necessary and sufficient for the existence social choice rules which are continuous, anonymous and respect unanimity (Chichilnisky 1980,1982), Theorem 13. The success of all four forms of resource allocation, by financial and real competitive markets, by cooperative games and by social choice, hinges on precisely the same limitation on the social diversity of the economy.

Shifting the angle of inquiry slightly sheds a different light on the subject. The results predict that a society which allocates resources efficiently by markets, collective choices or cooperative games, must exhibit no more than a certain degree of social 
diversity. This is an implicit prediction about the characteristics of those societies which implement successfully these forms of resource allocation. Increases in social diversity beyond this threshold may call for forms of resource allocation which are different from all those which are used today.

The results of this paper are intuitively clear. New forms of resource allocation appear to be needed in order to organize effectively a diverse society. But the issue is largely avoided by thinkers and policy makers alike because the institutions required for this do not yet exist, creating an uncomfortable vacuum. This paper attempts to formalize the problem within a rigorous framework and so provide a solid basis for theory and policy.

As defined here social diversity comes in many "shades", of which limited arbitrage is only one. The whole concept of social diversity is subtle and complex. It is encapsuled in an algebraic object, a family of cohomology rings ${ }^{8}$ denoted $C H$, which are naturally associated with a family of cones defined from the endowments and preferences of the traders in the economy. Limited arbitrage simply measures whether the cones intersect or not, while the rings $C H$ measure this and more: $C H$ reveal the intricate topology of how these cones are situated with respect to each other. The cohomology rings $\mathrm{CH}$ give a topological invariant of the economy, in the sense that $\mathrm{CH}$ is invariant under continuous deformations of the measurement of commodities. It is also structurally stable, remaining invariant under small errors of measurement. This concept of diversity is therefore ideal for the social sciences where measurements are imprecise and difficult to obtain. The properties of $\mathrm{CH}$ predict specific properties of the economy such as which subeconomies have a competitive equilibrium and which do not, which have a social choice rule and which do not, which have a core, and which have a supercore, Theorem 8 . The latter concept, the supercore, measures the extent of social cohesion, namely the extent to which a society has reasons to stay together or break apart. I prove that, somewhat paradoxically, the mildest form of social diversity predicts whether the supercore exists, even in economies where the preferences may not be convex.

The results presented here have two distinguishing features. One is that they provide a minimal condition which ensures that an Arrow Debreu equilibrium, ${ }^{9}$ the core and social choice rules exist, namely a condition which is simultaneously necessary and sufficient for the existence of solutions to each of these three forms of resource allocation. The second is they extend and unify the Arrow Debreu formulation of markets to encompass economies with or without short sales ${ }^{10}$ and with finitely or infinitely many markets.

While sufficient conditions for the existence of a competitive equilibrium have been known for about forty years, starting from the works of Von Neumann, Nash, Arrow and Debreu, the study of necessary and sufficient for resource allocation introduced in Chichilnisky (1991, 1992, 1993c,1994a,b,c, 1995) had been neglected previously. A necessary and sufficient condition is a useful tool. As an illustration consider the necessary and sufficient ("first order") conditions for partial equilibrium analysis of convex problems. These are among the most widely used tools in economics: they 
identify and help compute solutions in the theories of the consumer and of the firm, and in optimal growth theory. Equally useful could be a necessary and sufficient condition for the existence of market clearing allocations. Furthermore, in order to prove the equivalence between different problems of resource allocation one needs "tight" characterizations: a necessary and sufficient condition for equilibrium, the core and social choice is needed to establish the equivalence of these different forms of resource allocation.

It seems useful to elaborate on a geometric interpretation of limited arbitrage because it clarifies its fundamental links with the problem of resource allocation. It was recently established that the non-empty intersection of the cones which defines limited arbitrage is equivalent to a topological condition on the spaces of preferences (Chichilnisky 1980a, 1993c). The topological condition is contractibility, a form of similarity of preferences ${ }^{11}$ (Chichilnisky 1980, Heal 1983). Contractibility is necessary and sufficient for the existence of social choice rules, see Chichilnisky and Heal (1983). It turns out that the equivalence between non-empty intersection and contractibility is the link between markets and social choices. The contractibility of the space of preferences is necessary and sufficient for the existence of social choice rules, while non-empty intersection (limited arbitrage) is necessary and sufficient for the existence of a market equilibrium. One main result brings all this together: a family of convex sets has a non-empty intersection if and only if every subfamily has a contractible union, see Chichilnisky $(1980 \mathrm{a}, 1993) .{ }^{12}$

Using similar topological results, ${ }^{13}$ Theorem 6 establishes a link between the number of traders and the number of commodities: the economy has limited arbitrage if and only if every subeconomy of $N+1$ traders does, where $N$ is the number of commodities traded in the market.

As already mentioned, I consider economies with or without short sales: net trades are either bounded below, as in a standard Arrow Debreu economy, or they are not bounded at all. This is a considerable extension from the Arrow Debreu theory, as it includes financial markets in which short trades typically occur. ${ }^{14}$ In addition, the economy could have finitely or infinitely many markets: the results obtain in either case $^{15}$, Theorem 3.

It is somewhat surprising that the same condition of limited arbitrage is necessary and sufficient for the existence of a market equilibrium with or without short sales (Theorem 2). ${ }^{16}$ The non-existence of a competitive equilibrium is seemingly a different phenomenon in economies with short sales than it is in economies without short sales. With short sales, the problem of non-existence arises when traders with very different preferences ${ }^{17}$ desire to take unboundedly large positions against each other, positions which cannot be accommodated within a bounded economy. Instead, without short sales, the problem arises when some traders have zero income. Yet I show that in both cases the source of the problem is indeed the same: the diversity of the traders leads to ill-defined demand behavior at the potential market clearing prices, and prevents the existence of a competitive equilibrium. Limited arbitrage ensures that none of these problems arises: with or without short sales it bounds the diversity 
of traders precisely as needed for a competitive equilibrium to exist. Theorem 3 links the number of markets with the number of traders in a somewhat unexpected manner.

It is also somewhat surprising that the same condition of limited arbitrage ensures the existence of an equilibrium in economies with either finitely or infinitely many markets. The problem of existence appears to be different in these two cases, and indeed they are treated quite differently in the literature. A typical problem in economies with infinitely many markets is that positive orthants have empty interior, so that the Hahn-Banach theorem cannot be used to find equilibrium prices for efficient allocations. ${ }^{18}$ A solution to this problem was found in 1980: (Chichilnisky and Kalman, 1980) extended the Hahn-Banach theorem by introducing a cone condition and proving that it is necessary and sufficient for supporting convex sets whether or not they have an interior. Thereafter the cone condition has been used extensively to prove existence in economies with infinitely many markets and is by now a standard condition on preferences defined on infinitely many markets, known also under the name of "properness" of preferences in subsequent work. ${ }^{19}$ The fundamental new fact presented here is that limited arbitrage implies the cone condition on efficient and affordable allocations, Theorem $3 .^{20}$ Therefore by itself limited arbitrage provides a unified treatment of economies with finitely and infinitely many markets, being necessary and sufficient for the existence of equilibrium and the core in all cases.

In a nutshell: in all cases limited arbitrage bounds gains from trade, Proposition 4, and is equivalent to the compactness of the set of Pareto efficient utility allocations, Theorem 1. ${ }^{21}$ Gains from trade and the Pareto frontier are fundamental concepts involved in most forms of resource allocation: in markets, in games and in social choice. Limited arbitrage controls them all.

\section{Definitions and Examples}

An Arrow Debreu market $\mathrm{E}=\left\{X, \Omega_{h}, u_{h}, h=1, \ldots, H\right\}$ has $H \geq 2$ traders, indexed $h=1, \ldots, H, N \geq 2$ commodities and consumption or trading space ${ }^{22} X=R_{+}^{N}$ or $X=R^{N} ;$ in Section $5 X$ is a Hilbert space of infinite dimension. The vector $\Omega_{h} \in R_{+}^{N}$ denotes trader $h$ 's property rights or initial endowment and $\Omega=\left(\sum_{h=1}^{H} \Omega_{h}\right)$ is the total endowment of the economy; when $X=R_{+}^{N}, \Omega>0 .^{23}$ Traders may have zero endowments of some goods. Each trader $h$ has a continuous and convex preference represented by $u_{h}: X \rightarrow R$. This paper treats in a unified way general convex preferences where the normalized gradients define either an open or a closed map $x \rightarrow D u(x) /\|D u(x)\|$ on every indifference surface, so that either (i) all indifference surfaces contain no half lines or (ii) the normalized gradients to any closed set of indifferent vectors define a closed set. Some traders may have preferences of one type, and some of the other. Case (i) includes strictly convex preferences, and case (ii) linear preferences. All the assumptions and the results in this paper are ordinal; ${ }^{24}$ therefore without loss of generality one normalizes utilities so that for all $h, u_{h}(0)=0$ and $\sup _{\{x: x \in X\}} u_{h}(x)=\infty$. Preferences are increasing, i.e. $x>y \Rightarrow u_{h}(x) \geq u_{h}(y)$. 
When $X=R_{+}^{N}$ either indifference surfaces of positive utility are contained in the interior of $X, R_{++}^{N}$, such as Cobb-Douglas utilities, or if an indifference surface of positive utility intersects a boundary ray, it does so transversally. ${ }^{25}$

Definition 1 A preference is uniformly non-satiated when it is represented by a utility $u_{h}$ with a bounded rate of increase, ${ }^{26}$ e.g. for smooth preferences: $\exists \varepsilon, K>0$ : $\forall x \in X . K>\left\|D u_{h}(x)\right\|>\varepsilon$.

Uniformly non-satiated preferences are rather common: for example, preferences represented by linear utilities are uniformly non-satiated. The condition is a generalization of a standard Liftschitz condition.

Proposition 1 If a utility function $u_{n}: R^{N} \rightarrow R$ is uniformly non-satiated its indifference surfaces are within uniform distance from each other, i.e. $\forall r, s \in R, \exists N(r, s) \in$ $R$ such that $x \in u_{h}^{-1}(r) \Rightarrow \exists y \in u_{h}^{-1}(s)$ with $\|x-y\| \leq N(r, s)$.

Proof. This is immediate from the definition.

The preference in Figure 1 is not uniformly nonsatiated.

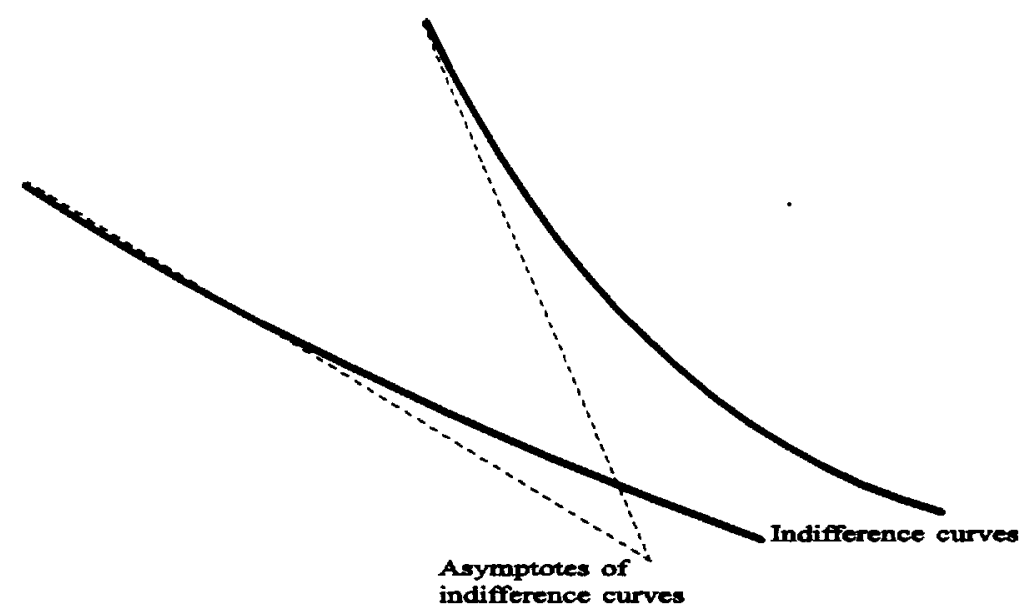

Figure 1. This preference is not uniformly nonsatiated because two indifference surfaces spread apart forever

\section{Assumption 1. When $X=R^{N}$, the preferences in the economy $\mathrm{E}$ are uniformly non-satiated.}

This includes preferences which are strictly convex or not, preferences whose indifference surfaces of positive utility intersect the boundary or not, and preferences whose indifference surfaces contain half lines or not, and are bounded below or not. Figure 2 illustrates. 


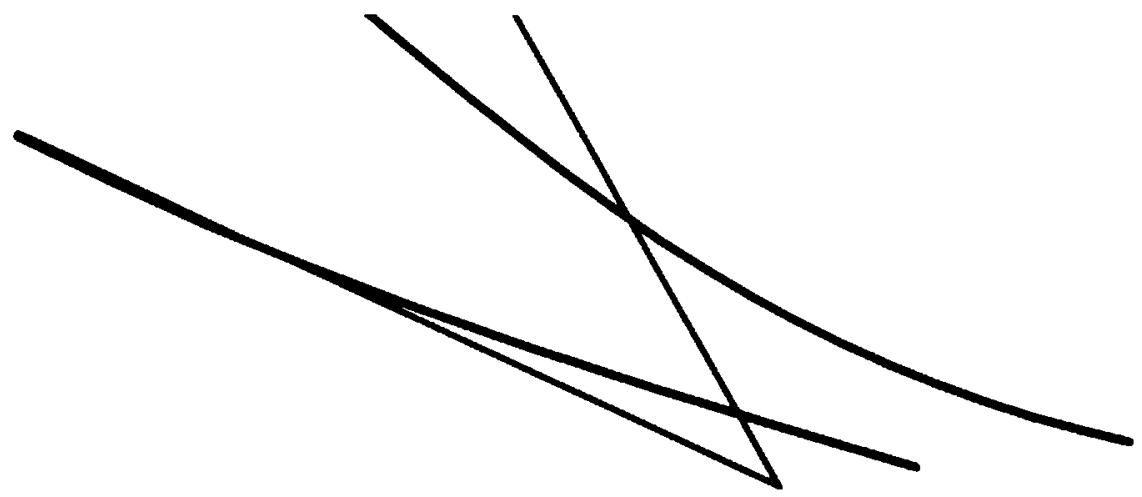

Figure 2. This preference is uniformly nonsatiated

The space of feasible allocations is $\Upsilon=\left\{\left(x_{1}, \ldots, x_{H}\right) \in X^{H}: \sum_{h=1}^{H} x_{h}=\Omega\right\}$. The set of supports to individually rational affordable efficient resource allocations is:

$$
\begin{gathered}
S(\mathbf{E})=\left\{v \in R^{N}: \text { if }\left(x_{1} \ldots x_{H}\right) \in \Upsilon \text { with } u_{h}\left(x_{h}\right) \geq u_{h}\left(\Omega_{h}\right) \forall h=1, \ldots H,\right. \\
\left.\left\langle v, x_{h}-\Omega_{h}\right\rangle=0, \text { then } u_{h}\left(z_{h}\right) \geq u_{h}\left(x_{h}\right) \forall h \text { implies }\left\langle v, z_{h}-x_{h}\right\rangle \geq 0\right\} .
\end{gathered}
$$

The set of prices orthogonal to the endowments is ${ }^{27}$

$$
N=\left\{v \in R_{+}^{N}-\{0\}: \exists h \text { with }\left\langle v, \Omega_{h}\right\rangle=0\right\} .
$$

The utility possibility set of the economy $E$ is the set of feasible and individually rational utility allocations:

$$
\begin{gathered}
U(\mathrm{E})=\left\{\left(V_{1}, \ldots, V_{H}\right): \forall h, V_{h}=u_{h}\left(x_{h}\right) \geq u_{h}\left(\Omega_{h}\right) \geq 0,\right. \\
\text { for some } \left.\left(x_{1}, \ldots, x_{H}\right) \in \Upsilon\right\}
\end{gathered}
$$

The Pareto frontier of the economy $E$ is the set of feasible, individually rational and efficient utility allocations:

$$
P(\mathrm{E})=\{V \in U(\mathrm{E}): \sim \exists W \in U(\mathrm{E}): W>V\} \subset R_{+}^{H} .
$$

A competitive equilibrium of $E$ consists of a price vector $p^{*} \in R_{+}^{N}$ and an allocation $\left(x_{1}^{*} \ldots x_{H}^{*}\right) \in X^{H}$ such that $x_{h}^{*}$ optimizes $u_{h}$ over the budget set $B_{h}\left(p^{*}\right)=\{x \in X$ : $\left.\left\langle x, p^{*}\right\rangle=\left\langle\Omega_{h}, p^{*}\right\rangle\right\}$ and $\sum_{h=1}^{H} x_{h}^{*}-\Omega_{h}=0$.

\subsection{Global and Market Cones}

The global cones defined here are identical to those introduced in Chichilnisky (1995); the notation is adapted to the context. Two cases, $X=R^{N}$ and $X=R_{+}^{N}$, are considered separately.

- Consider first $X=R^{N}$. 
Definition 2 For trader $h$ define the global cone of directions along which utility increases without bound:

$$
A_{h}\left(\Omega_{h}\right)=\left\{x \in X: \forall y \in X, \exists \lambda>0: u_{h}\left(\Omega_{h}+\lambda x\right)>u_{h}(y)\right\}
$$

This cone contains global information on the economy. ${ }^{28}$ In ordinal terms, the rays of this cone intersect all indifference surfaces corresponding to bundles preferred by $u_{h}$ to $\Omega_{h}$. This cone and the part of its boundary along which utility never ceases to increase define:

$$
G_{h}\left(\Omega_{h}\right)=\left\{x \in X: \sim \exists \operatorname{Max}_{\lambda \geq 0} u_{h}\left(\Omega_{h}+\lambda x\right)\right\}
$$

This cone is identical to the global cone of Chichilnisky (1995), p. $85,(4) ;^{29}$ the current definition treats all convex preferences in a unified way. Under Assumption 1 $G_{h}\left(\Omega_{h}\right)$ has a simple structure: when preferences have half lines in their indifferences $G_{h}\left(\Omega_{h}\right)$ equals $A_{h}\left(\Omega_{h}\right)$; when indifferences contain no half lines, then $G_{h}\left(\Omega_{h}\right)$ is its closure, see also Chichilnisky 1995, p. 85.

Definition 3 The market cone of trader $h$ is

$$
D_{h}\left(\Omega_{h}\right)=\left\{z \in X: \forall y \in G_{h}\left(\Omega_{h}\right),\langle z, y\rangle>0\right\}
$$

$D_{h}$ is the cone of prices assigning strictly positive value to all directions of net trades leading to eventually increasing utility. This is a convex cone.

The following proposition establishes the structure of the global cones, and is used in proving the connection between limited arbitrage, equilibrium and the core:

Proposition 2 If the function $u_{h}: R^{N} \rightarrow R$ is uniformly non-satiated: (i) The interior of the global cone is

$$
\begin{aligned}
G_{h}^{o}\left(\Omega_{h}\right) & =A_{h}^{o}\left(\Omega_{h}\right) \\
& =\left\{z \in G_{h}\left(\Omega_{h}\right): \lim _{\lambda \rightarrow \infty} u_{h}\left(\Omega_{h}+\lambda z\right)=\infty\right\} \neq \emptyset .
\end{aligned}
$$

(ii) The boundary of the cone $G_{h}\left(\Omega_{h}\right), \partial G_{h}\left(\Omega_{h}\right)$, contains (a) those directions along which utility increases towards a bounded value that is never reached:

$$
B_{h}\left(\Omega_{h}\right)=\left\{z \in \partial A_{h}\left(\Omega_{h}\right): \forall \lambda>0, u_{h}\left(\Omega_{h}+\lambda z\right) \neq \lim _{\lambda \rightarrow \infty} u_{h}\left(\Omega_{h}+\lambda z\right)<\infty\right\}
$$

and (b) those directions along which the utility eventually achieves a constant value:

$$
C_{h}\left(\Omega_{h}\right)=\left\{z \in \partial A_{h}\left(\Omega_{h}\right): \exists N: \lambda, \mu>N \Rightarrow u_{h}\left(\Omega_{h}+\lambda z\right)=u_{h}\left(\Omega_{h}+\mu z\right)\right\},
$$

(iii) the interior of the global cone, its boundary and its closure and the cones $G_{h}$ and $D_{h}$ are uniform across all vectors in the space, i.e. $\forall \Omega, \Lambda \in X$ :

$$
\begin{aligned}
G_{h}^{o}(\Omega) & =G_{h}^{o}(\Lambda)=A_{h}^{o} \\
B_{h}(\Omega) \cup C_{h}(\Omega) & \subset \partial G_{h}(\Omega)=\partial G_{h}(\Lambda)=\partial G_{h} \\
G_{h}(\Omega) & =G_{h}(\Lambda)=G_{h}, D_{h}(\Omega)=D_{h}(\Lambda)=D_{h}
\end{aligned}
$$

and in particular

$$
\bar{G}_{h}(\Omega)=\bar{G}_{h}(\Lambda)=\bar{G}_{h}
$$


(iv) For general non-satiated preferences $G_{h}\left(\Omega_{h}\right)$ and $D_{h}\left(\Omega_{h}\right)$ may not be uniform. Proof. The three sets $A_{h}\left(\Omega_{h}\right), B_{h}\left(\Omega_{h}\right)$ and $C_{h}\left(\Omega_{h}\right)$ are disjoint pairwise and

$$
A_{h}\left(\Omega_{h}\right) \cup B_{h}\left(\Omega_{h}\right) \cup C_{h}\left(\Omega_{h}\right) \cup H_{h}\left(\Omega_{h}\right)=R^{N} .
$$

where $H_{h}\left(\Omega_{h}\right)$ is the complement of $A_{h}\left(\Omega_{h}\right) \cup B_{h}\left(\Omega_{h}\right) \cup C_{h}\left(\Omega_{h}\right)$, i.e. the set of directions along which the utility achieves a maximum value and decreases thereafter.

The first step is to show that $B_{h}\left(\Omega_{h}\right) \cup C_{h}\left(\Omega_{h}\right) \subset \partial G_{h}\left(\Omega_{h}\right)$. Observe that monotonicity and the condition of uniform non satiation imply that the rate of increase is uniformly bounded below along the direction defined by the vector $(1, \ldots, 1)$ (or along any direction defined by a strictly positive vector). This implies that if $z \in$ $B_{h}\left(\Omega_{h}\right) \cup C_{h}\left(\Omega_{h}\right)$

$$
s>>z \Rightarrow s \in A_{h}\left(\Omega_{h}\right)
$$

and

$$
s<<\Rightarrow s \in H_{h}\left(\Omega_{h}\right)
$$

Therefore the sets $B_{h}\left(\Omega_{h}\right) \cup C_{h}\left(\Omega_{h}\right)$ is in the boundary of the set $A_{h}\left(\Omega_{h}\right)$. The relation between $G_{h}\left(\Omega_{h}\right)$ and $A_{h}\left(\Omega_{h}\right)$ is now immediate, cf. Chichilnisky (1995) p. 85, (4).

The next step is to show that $A_{h}\left(\Omega_{h}\right)$ is identical everywhere. It suffices to show that if two different half-lines $l=\left\{\Omega_{h}+\lambda v\right\}_{\lambda \geq 0}$ and $m=\left\{\Lambda_{h}+\lambda v\right\}_{\lambda \geq 0}$ are parallel translates of each other, and $l \subset A_{h}\left(\Omega_{h}\right)$, then $m \subset A_{h}\left(\Lambda_{h}\right), \forall \Lambda_{h} \in m$. This is immediate from Assumption 1, which ensures that the rate of increase of the function $u_{h}$ is bounded above: if the values of the function $u_{h}$ on $m$ were bounded above, while exceeding every bounded value over the (parallel) line $l$, then the rate of increase of the utility would be unbounded above.

$\mathrm{By}$ assumption, preferences either have half lines in their indifferences, or they don't: in either case the sets $B_{h}\left(\Omega_{h}\right)$ and $C_{h}\left(\Omega_{h}\right)$ are uniform. In addition, $A_{h}\left(\Omega_{h}\right)$ is uniform as well. Therefore to complete the proof it remains only to show that the cones $G_{h}\left(\Omega_{h}\right)$ are the same everywhere under Assumption 1 .

Observe that for a general convex preference represented by a utility $u_{h}$ the set $G_{h}\left(\Omega_{h}\right)$ may vary as the vector $\Omega_{h}$ varies, since the set $B_{h}\left(\Omega_{h}\right)$ itself may vary with $\Omega_{h}$ : at some $\Omega_{h}$ a direction $z \in \partial G_{h}$ may be in $B_{h}\left(\Omega_{h}\right)$ and at others $B_{h}\left(\Omega_{h}\right)$ may be empty and $z \in C_{h}\left(\Omega_{h}\right)$ instead. This occurs when along a ray defined by a vector $z$ from one endowment the utility levels asymptote to a finite limit but do not reach their limiting value, while at other endowments, along the same direction $z$, they achieve this limit. This example, and a similar reasoning for $A_{h}\left(\Omega_{h}\right)$, proves $(i v)$. However, such cases are excluded here, since under our assumptions on preferences, for each trader, either all indifference surfaces contain half lines, or none do. This completes the proof of the proposition. 


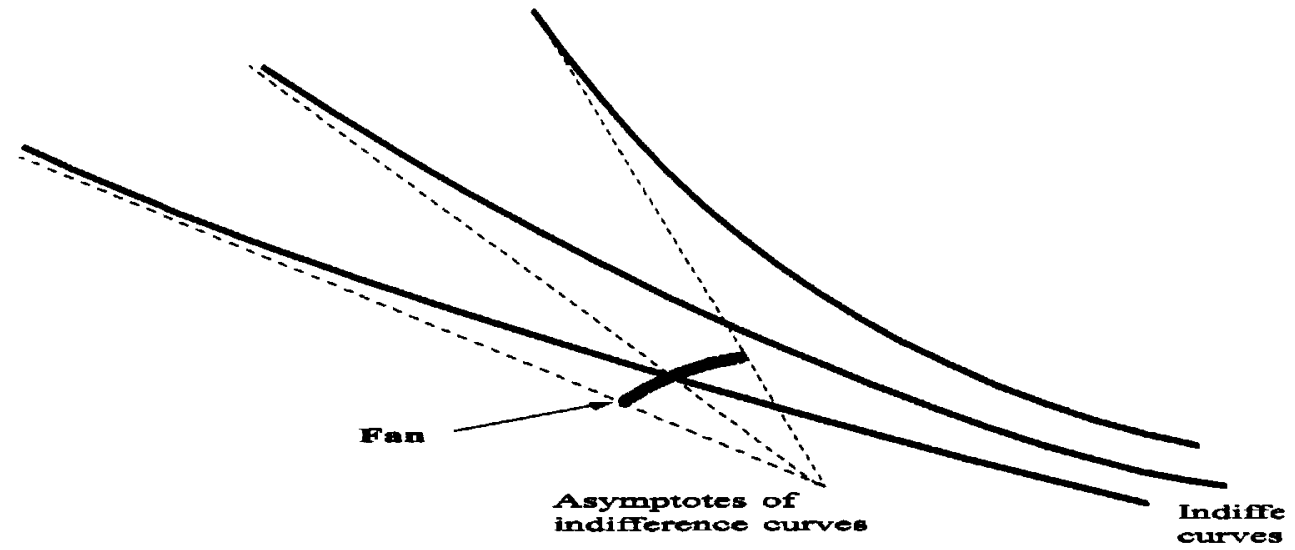

Figure 3. This preference has a 'fan' of different directions along which the utility values reach a bounded utility value. Assumption 1 is not satisfied. All the directions in the fan are in the recession cone but not in the global cone $G_{h}$ nor in the cone $A_{h}$.

- Consider next the case: $X=R_{+}^{N}$

Definition 4 The market cone of trader $h$ is:

$$
\begin{aligned}
D_{h}^{+}\left(\Omega_{h}\right) & =D_{h}\left(\Omega_{h}\right) \cap S(\mathrm{E}) \text { if } S(\mathrm{E}) \subset \mathrm{N} \\
& =D_{h}\left(\Omega_{h}\right) \text { otherwise. }
\end{aligned}
$$

where $S(\mathrm{E})$ and $\mathrm{N}$ are defined in (1) and (2). ${ }^{30}$

There is no analog to Proposition 2 when $X=R_{+}^{N}$; indeed, when $X=R_{+}^{N}$ the market cones $D_{h}^{+}\left(\Omega_{h}\right)$ typically vary with the initial endowments. However, when $\Omega_{h} \in R_{++}^{N}$, the interior of $R_{+}^{N}$, then $D_{h}^{+}\left(\Omega_{h}\right)=D_{h}\left(\Omega_{h}\right)$ and therefore $D_{h}^{+}\left(\Omega_{h}\right)$ is the same for all endowments in $R_{++}^{N}$.

Proposition 3 When $X=R_{+}^{N}$ and an indifference surface of $u_{h}$ corresponding to a positive consumption bundle $x>0$ intersects a boundary ray ${ }^{31} r \subset \partial X$, then $r \in G_{h}(0){ }^{32}$

Proof. Recall that we assumed $u_{h}(0)=0$, and that the preference's indifference surfaces of positive utility are either (a) contained in the interior of $R_{+}^{N}, R_{++}^{N}$, or (b) they intersect a boundary ray $r$ of $R_{+}^{N}$ and do so transversally. In case (a) the proposition is satisfied trivially, because no indifference surface of strictly positive value ever intersects the boundary of $R_{+}^{N}$. In case (b) the proposition follows immediately from the definition of transversality. Observe that it is possible that $\sup _{x \in r}\left(u_{h}(x)\right)<\infty$. 


\subsection{The Core and the Supercore}

Definition 5 The core of the economy $E$ is the set of allocations which no coalition can improve upon within its own endowments:

$$
\begin{gathered}
C(\mathrm{E})=\left\{\left(x_{1} \ldots . x_{H}\right) \in R^{N \times H}: \sum_{h}\left(x_{h}-\Omega_{h}\right)=0 \text { and } \sim J \subset\{1, \ldots H\}:\right. \\
\text { and }\left\{y_{h}\right\}_{h \in J} \text { s.t. } \sum_{j \in J}\left(y_{j}-\Omega_{j}\right)=0, \forall j \in J, u_{j}\left(y_{j}\right) \geq u_{j}\left(x_{j}\right), \\
\text { and } \left.\exists j \in J: u_{j}\left(y_{j}\right)>u_{j}\left(x_{j}\right)\right\} .
\end{gathered}
$$

Definition 6 The supercore of the economy is the set of allocations which no strict sub coalition can improve using only its own endowments. It is therefore a superset of the core:

$$
\begin{gathered}
S C(\mathrm{E})=\left\{\left\{\left(x_{1}, \ldots, x_{H}\right) \in R^{N \times H}: \sum_{h}\left(x_{h}-\Omega_{h}\right)=0 \text { and } \sim J \subset\{1, \ldots,\}\right]:\right. \\
J \neq\{1, \ldots, H\} \text { and }\left\{y_{h}\right\}_{h \in J} \text { s.t. } \forall j \in J, u_{j}\left(y_{j}\right) \geq u_{j}\left(x_{j}\right), \sum_{j \in J}\left(y_{j}-\Omega_{j}\right)=0, \text { and } \\
\left.\exists j \in J: u_{j}\left(y_{j}\right)>u_{j}\left(x_{j}\right)\right\} .
\end{gathered}
$$

By construction, $C(\mathrm{E}) \subset S C(\mathrm{E})$. The motivation for this concept is as follows: if an allocation is in the supercore, no strict subcoalition of traders can improve upon this by itself. A non-empty supercore means that no strict subsets of individuals can do better than what they can do by joining the entire group. The benefits from joining the larger group exceed those available to any subgroup. One can say therefore that an economy with a non-empty supercore has reasons to stay together: There is no reason for such a society to break apart. If an economy that has stayed together for some time, it probably has a non-empty supercore.

\section{Limited Arbitrage: Definition and Examples}

This section provides the definition of limited arbitrage. It gives an intuitive interpretation for limited arbitrage in terms of gains from trade, and contrasts limited arbitrage with the arbitrage concept used in financial markets. It provides examples of economies with and without limited arbitrage.

Definition 7 When $X=R^{N}$, E satisfies limited arbitrage when

$$
\text { (LA) } \bigcap_{h=1}^{H} D_{h} \neq \emptyset .
$$

Definition 8 When $X=R_{+}^{N}$, E satisfies limited arbitrage when 


$$
\left(L A^{+}\right) \bigcap_{h=1}^{H} D_{h}^{+}\left(\Omega_{h}\right) \neq \emptyset .
$$

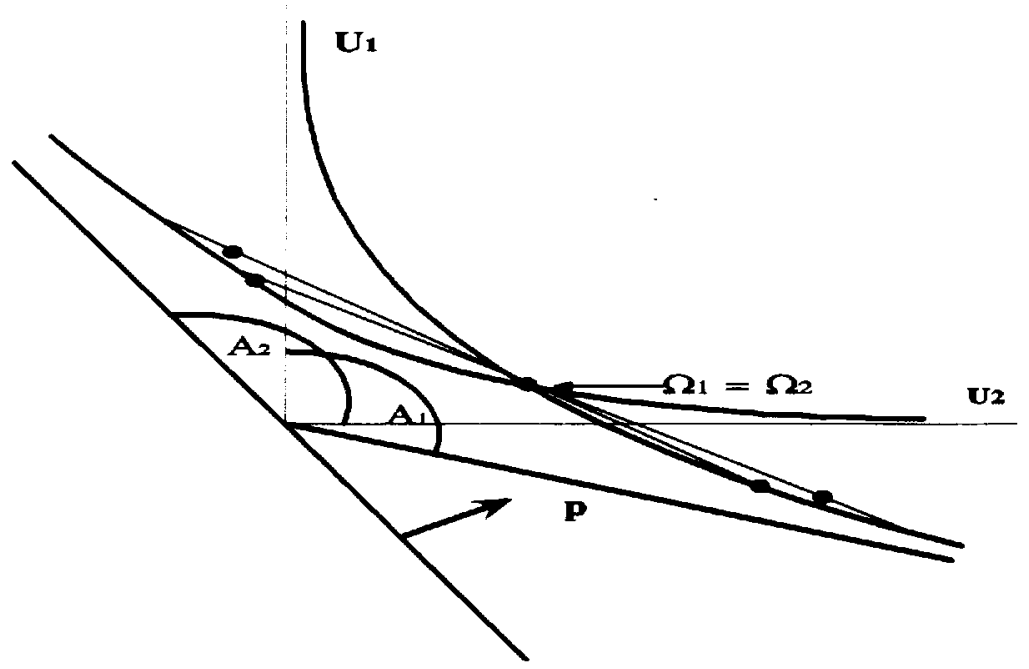

Figure 4. Limited arbitrage is satisfied: feasible allocations lead to bounded utility increases.

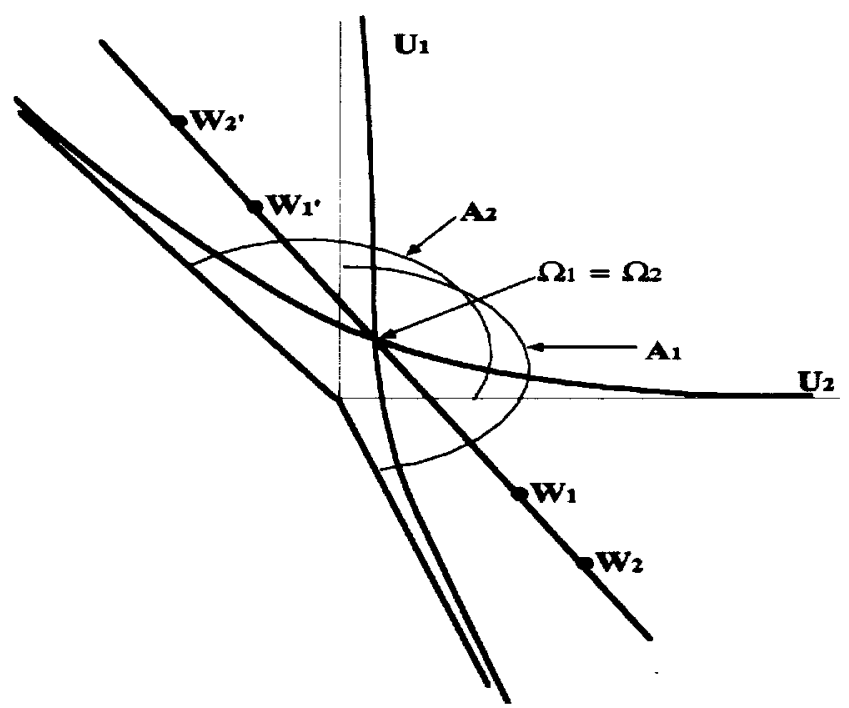

Figure 5. Limited arbitrage is not satisfied: there exist a feasible unbounded sequence of allocations, $\left(W_{1}, W_{1}^{\prime}\right),\left(W_{2}, W_{2}^{\prime}\right), \ldots$, along which both traders' utility never ceases to increase. 


\subsection{Interpretation of Limited Arbitrage as Bounded Gains From Trade when $X=R^{N}$}

Limited arbitrage has a simple interpretation in terms of gains from trade when $X=R^{N}$. Gains from trade are defined by:

$$
\begin{gathered}
\mathrm{G}(\mathrm{E})=\sup \left\{\sum_{h=1}^{H}\left(u_{h}\left(x_{h}\right)-u_{h}\left(\Omega_{h}\right)\right\},\right. \text { where } \\
\sum_{h=1}^{H}\left(x_{h}-\Omega_{h}\right)=0, \text { and } \forall h, u_{h}\left(x_{h}\right) \geq u_{h}\left(\Omega_{h}\right) \geq 0 .
\end{gathered}
$$

The Proposition below applies to preferences where the normalized gradients define a closed map on every indifference surface, i.e. case (ii); the Corollary following it applies both to case (i) and (ii):

Proposition 4 In case (ii), the economy $\mathrm{E}$ satisfies limited arbitrage if and only if gains from trade are bounded ${ }^{33}$ i.e. if and only if

$$
\mathrm{G}(\mathrm{E})<\infty \text {. }
$$

Proof. Assume $E$ has limited arbitrage. If $\mathrm{G}(\mathrm{E})$ was not bounded there would exist a sequence of net trades $\left(z_{1}^{j} \ldots z_{H}^{j}\right)_{j=1,2 \ldots}$ such that (i) $\forall j, \sum_{h=1}^{H} z_{h}^{j}=0, \forall h, j u_{h}\left(z_{h}^{j}\right) \geq 0$, and (ii) for some $h=g, \lim _{j \rightarrow \infty}\left(u_{g}\left(\Omega_{g}+z_{g}^{j}\right)\right) \rightarrow \infty$. Next I will show that if $\left\|z_{h}^{j}\right\| \rightarrow \infty$, and $\left\{z_{h}^{j} /\left\|z_{h}^{j}\right\|\right\}_{j=1,2, \ldots}$ denotes a convergent subsequence, then $z_{h}=\lim _{j} z_{h}^{j} /\left\|z_{h}^{j}\right\| \epsilon$ $\bar{G}_{h}$. The proof is by contradiction. By Proposition 2 the cone $\bar{G}_{h}$ is uniform so without loss of generality we may assume that $\forall h \Omega_{h}=0$. If $z_{h} \in \bar{G}_{h}^{c},{ }^{34}$ then by quasiconcavity of $u_{h}$ and by Proposition 2, along the ray defined by $z_{h}$ the utility $u_{h}$ achieves a maximum level $u^{o}$, say at $\lambda_{o} z$, for some $\lambda_{o} \geq 0$, and it decreases thereafter, i.e. $\lambda>$ $\lambda_{o} \Rightarrow u_{h}(\lambda z)<u^{o}$. Define a function $\theta: R_{+} \rightarrow R_{+}$by $u_{h}\left(\lambda z_{h}+\theta(\lambda) e\right)=u^{o}$, where $e=(1, \ldots, 1)$. I will show that $\theta$ is a convex function so necessarily $\lim _{\lambda \rightarrow \infty} \theta(\lambda)=\infty$. By convexity of preferences

$$
\begin{gathered}
u^{o} \leq u_{h}\left(\alpha\left(\lambda z_{h}+\theta(\lambda) e\right)+(1-\alpha)\left(\lambda^{\prime} z_{h}+\theta\left(\lambda^{\prime}\right) e\right)\right. \\
=u_{h}\left(\left(\alpha \lambda+(1-\alpha) \lambda^{\prime}\right) z_{h}+\left(\alpha \theta(\lambda)+(1-\alpha)\left(\theta\left(\lambda^{\prime}\right)\right) e\right) .\right.
\end{gathered}
$$

Thus by monotonicity and by the definition of the map $\theta, \theta\left(\alpha \lambda+(1-\alpha) \lambda^{\prime}\right) \leq$ $\alpha \theta(\lambda)+(1-\alpha) \theta\left(\lambda^{\prime}\right)$, which proves convexity. So necessarily $\lim _{\lambda \rightarrow \infty} \theta(\lambda)=\infty$.

Assumption 1 together with monotonicity implies that the rate of increase of $u_{h}$ along the direction defined by $e$ (or by any strictly positive vector) is uniformly bounded below: $\exists \varepsilon>0:\left|u_{h}(x+\theta e)-u_{h}(x)\right| \geq \theta . \varepsilon, \forall \theta \in R_{+}, \forall x \in R^{N}$. Therefore $u_{h}\left(\lambda z_{h}+\theta(\lambda) e\right) \equiv u^{o} \geq u_{h}\left(\lambda z_{h}\right)+\theta(\lambda) \varepsilon$, so that $u_{h}\left(\lambda z_{h}\right) \leq u^{o}-\theta(\lambda) e$. Note that $\theta\left(\lambda_{o}\right)=0$ and $\theta(\lambda)>0$ for $\lambda>\lambda_{o}$. I showed above that $\theta$ is a convex function. Therefore $\lim _{\lambda \rightarrow \infty} \theta(\lambda)=\infty$; since $u_{h}\left(\lambda z_{h}\right) \leq u^{\circ}-\theta(\lambda)$ e then $\lim _{\lambda \rightarrow \infty} u_{h}\left(\lambda z_{h}\right)=-\infty$. 
It follows that $z_{h} \in \bar{G}_{h}$ for otherwise as we have seen $\lim _{j \rightarrow \infty} u_{h}\left(z_{h}^{j}\right)<0$ contradicting the fact that the utility levels of $\left(z_{1}^{j}, \ldots, z_{H}^{j}\right)_{j=1,2 \ldots}$ are positive.

Recall that for some $g, \lim _{j \rightarrow \infty} u_{g}\left(z_{g}^{j}\right) \rightarrow \infty$. By Assumption 1, $\exists K>0: \mid u_{g}(x)-$ $u_{g}(y) \mid \leq K\|x-y\| \forall x, y \in R^{N}$. so that for any $n$ and $j\left|u_{g}\left(z_{g}^{n}\right)-u_{g}\left(z_{g}^{n}-j e\right)\right| \leq K \|$ je $\|$. Since $u_{g}\left(z_{g}^{j}\right) \rightarrow \infty$, for every $j$ there exists an $n_{j}$ such that $u_{g}\left(z_{g}^{n_{j}}-j e\right)>j$. Take the sequence $\left\{z_{g}^{n_{j}}\right\}$ and relabel it $\left\{z_{g}^{j}\right\}$. Now consider the new sequence of allocations $\left\{z_{1}^{j}+\frac{j \epsilon}{H-1}, \ldots, z_{g}^{j}-j e, \ldots, z_{H}^{j}+\frac{j e}{H-1}\right\}$ and call it also $\left\{z_{h}^{j}\right\}_{h=1,2, \ldots, H}$. For each $j$ this defines a feasible allocation and, by Assumption 1, along this sequence $\forall h, u_{h}\left(z_{h}^{j}\right) \rightarrow \infty$. In particular $\forall h .\left\|z_{h}^{j}\right\| \rightarrow \theta$.

Define now $C$ as the set of all strictly positive convex combinations of the vectors $z_{h}=\lim _{j} z_{h}^{j} /\left\|z_{h}^{j}\right\|$ for all $h$. Then either $C$ is strictly contained in a half space, or it defines a subspace of $R^{N}$. Since $\sum_{h=1}^{H} z_{h}^{j}=0, C$ cannot be strictly contained in a half space. Therefore $C$ defines a subspace. In particular for any given $g, \exists \lambda_{h} \geq 0$ $\forall h$ such that $\left(^{*}\right)-z_{g}=\sum_{h=1}^{H} \lambda_{h} z_{h}$. If one trader had indifference surfaces without half lines (case (i)) then $G_{g}=\bar{G}_{g}$ and $z_{g} \in \bar{G}_{g} \Rightarrow z_{g} \in G_{g}$, so that limited arbitrage would contradict $\left({ }^{*}\right)$, because there can be no $p$ such that $\langle p, x\rangle>0$ for $x \in \bar{G}_{h}$ and $\langle p, x\rangle>0$ for $x \in G_{g}$. When instead for every closed sequence of indifferent vectors the corresponding normals define a closed set, i.e. all preferences are in case (ii), then the global cone $G_{h}$ is open (Chichilnisky (1995)) so that $G_{h}^{c}$ is a closed set, and the set of directions in $G_{h}^{c}$ is compact. On each direction of $G_{h}^{c}$ the utility $u_{h}$ achieves a maximum by definition; therefore under the conditions on preferences there exists a maximum utility level for $u_{h}$ over all directions in $G_{h}^{c}$. Since along the sequence $\left\{z_{h}^{j}\right\}$ every trader's utility increases without bound, $\forall h \exists j_{h}: j>j_{h} \Rightarrow z_{h}^{j} \in G_{h}$. However $\sum_{h=1}^{H} z_{h}^{j}=0$, contradicting again limited arbitrage. In all cases the contradiction arises from assuming that $G(E)$ is not bounded, so that $G(E)$ must be bounded. Therefore under Assumption 1, limited arbitrage implies bounded gains from trade. Observe that when all preferences are in case (ii) then $G_{h}=A_{h}$. In this case the reciprocal is immediate: limited arbitrage is also necessary for bounded gains from trade, completing the proof.

The proof of the sufficiency in Proposition 4 above is valid for all preferences satisfying Assumption 1, case (i) or case (ii), so that:

Corollary 1 For all economies with uniformly non-satiated preferences, limited arbitrage implies bounded gains from trade. ${ }^{33}$

\subsection{A Financial Interpretation of Limited Arbitrage}

It is useful to explain the connection between limited arbitrage and the notion of "no-arbitrage" used in finance. The concepts are generally different, but in certain cases they coincide. In the finance literature, arbitrage appears as a central concept. Financial markets equilibrium is often defined as the absence of market arbitrage. In Walrasian markets this is not the case. It may therefore appear that the two literatures use different equilibrium concepts. Yet the link provided here draws a 
bridge between these two literatures. As shown below limited arbitrage, while not an equilibrium concept, is necessary and sufficient for the existence of a Walrasian equilibrium. In the following I will show the close link between the two concepts and establish the bridge between the two equilibrium theories. I will provide examples where the two concepts are identical, and others where they are different.

In financial markets an arbitrage opportunity exists when unbounded gains can be made at no cost, or, equivalently, by taking no risks. Consider, for example, buying an asset in a market where its price is low while simultaneously selling it at another where its price is high: this can lead to unbounded gains at no risk to the trader. No-arbitrage means that such opportunities do not exist, and it provides a standard framework for pricing a financial asset: precisely so that no arbitrage opportunities should arise between this and other related assets. Since trading does not cease until all arbitrage opportunities are extinguished, at a market clearing equilibrium there must be no-arbitrage.

The simplest illustration of the link between limited arbitrage and no-arbitrage is an economy $\mathbf{E}$ where the traders' initial endowments are zero, $\Omega_{h}=0$ for $h=1,2$, and the set of gradients to indifference surfaces are closed. Here no-arbitrage at the initial endowments means that there are no trades which could increase the traders' utilities at zero cost: gains from trade in $E$ must be zero. By contrast, $E$ has limited arbitrage when no trader can increase utility beyond a given bound at zero cost; as seen above, gains from trade are bounded.

In brief: no-arbitrage requires that there should be no gains from trade at zero cost while limited arbitrage requires that there should be only bounded utility arbitrage or limited gains from trade.

Now consider a particular case of the same example: when the traders' utilities are defined by linear real valued functions. Then the two concepts coincide: there is limited arbitrage if and only if there is no-arbitrage as defined in finance. In brief: in linear economies, limited arbitrage "collapses" into no-arbitrage.

In general, the two concepts are related but nonetheless different: no-arbitrage is a market clearing condition used to describe an allocation at which there is no further reason to trade. It can be applied at the initial allocations, but then it means that there is no reason for trade in the economy as a whole: the economy is autarchic and therefore not very interesting. By contrast, limited arbitrage is applied only to the economy's initial data, the traders' endowments and preferences. Limited arbitrage does not imply that the economy is autarchic; quite to the contrary, it is valuable in predicting whether the economy can ever reach a competitive equilibrium. It allows to do so by examining the economy's initial conditions.

\subsection{Examples of markets with and without limited arbitrage}

Example 1 Figures 4 and 5 above illustrate an economy with two traders trading in $X=R^{2}$; in Figure 4 the market cones intersect and the economy has limited arbitrage. In Figure 5 the market cones do not intersect and the economy does not have limited arbitrage. Figure 6 below illustrates three traders trading in $X=R^{3}$; 
each two market cones intersect, but the three market cones do not intersect, and the economy violates limited arbitrage. This figure illustrates the fact that the union of the market cones may fail to be contractible: indeed, this failure corresponds to the failure of the market cones to intersect. as proven in Chichilnisky (1993c).

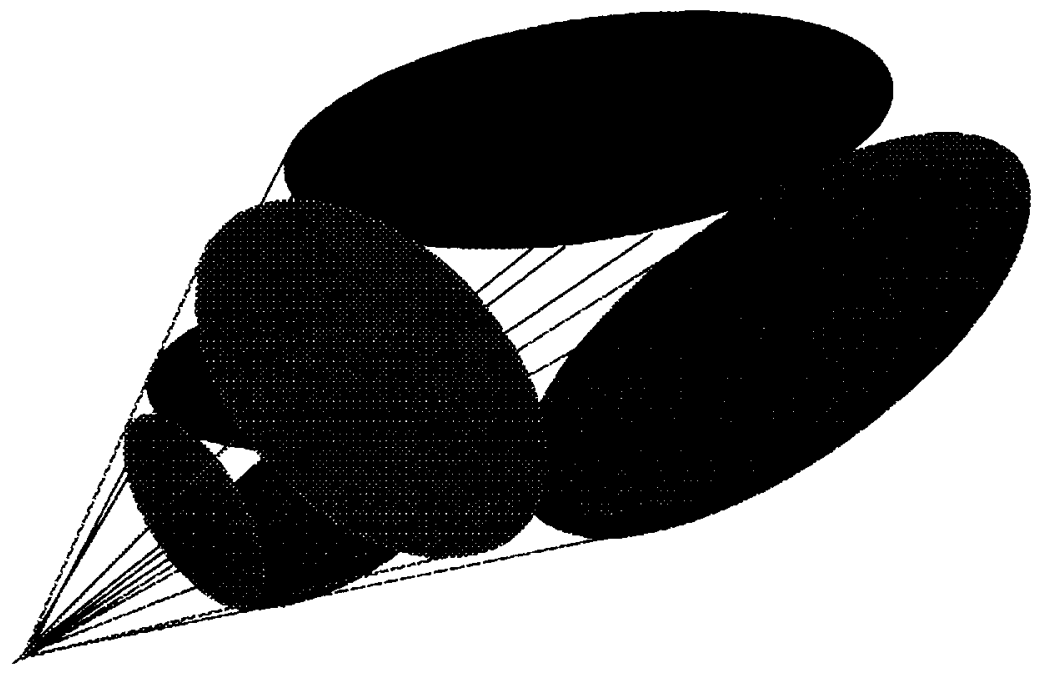

Figure 6. Three traders in $\mathrm{R}^{3}$. Every two traders's subeconomy has limited arbitrage but the whole economy does not.

Example 2 When the consumption set is $X=R_{+}^{N}$, limited arbitrage is always satisfied if all indifference surfaces through positive consumption bundles are contained in the interior of $X, R_{++}^{N}$. Examples of such preferences are those given by CobbDouglas utilities, or by utilities with constant elasticity of substitution (CES) with elasticity of substitution $\sigma<1$. This is because all such preferences have as global cone the positive orthant (or its closure), and therefore their market cones always intersect. These preferences are very similar to each other on choices involving large utility levels: this is a form of similarity of preferences. Economies where the individuals' initial endowments are strictly interior to the consumption set $X$ always satisfy the limited arbitrage condition in the case $X=R_{+}^{N}$, since in this case $\forall$, $R_{++}^{N} \subset D_{h}^{+}\left(\Omega_{h}\right)$ for all $h=1, \ldots, H$.

Example 3 When $X=R_{+}^{N}$ the limited arbitrage condition may fail to be satisfied when some trader's endowment vector $\Omega_{h}$ is in the boundary of the consumption space, $\partial R_{+}^{N}$, and at all supporting prices in $S(\mathrm{E})$ some trader has zero income, i.e. when $\forall p \in S(\mathrm{E}) \exists h$ such that $\left\langle p, \Omega_{h}\right\rangle=0$. In this case, $S(\mathrm{E}) \subset \mathrm{N}$. This case is illustrated in Figure 7 below; it is a rather general case which may occur in economies with many individuals and with many commodities. When all individuals have positive income at some price $p \in S(\mathrm{E})$, then limited arbitrage is always satisfied since by definition in this case $\forall h,=R_{++}^{N} \subset D_{h}^{+}\left(\Omega_{i}\right)$ for all $h=1, \ldots, H$. 


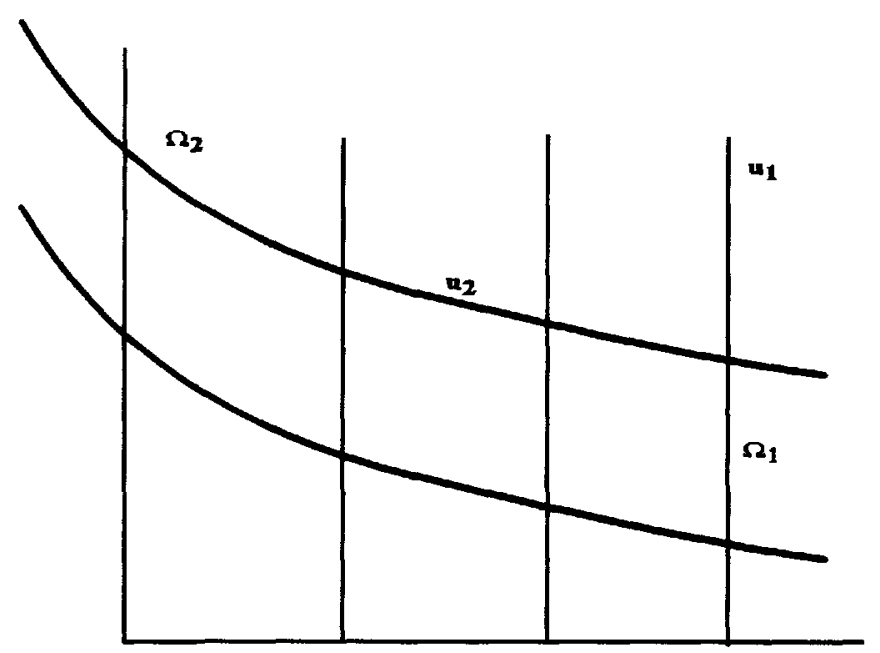

Figure 7. Limited arbitrage fails. Trader two owns only one good, to which the other trader is indifferent.

Example 4 A competitive equilibrium may exist even when some traders have zero income, showing that Arrow's "resource relatedness" condition (Arrow and Hahn (1971)) is sufficient but not necessary for existence of an equilibrium. Figure 8 below illustrates an economy where at all supporting prices some trader has zero income: $\forall p \in S(\mathrm{E}) \exists h$ such that $\left\langle p, \Omega_{h}\right\rangle=0$, i.e. $S(\mathrm{E}) \subset \mathrm{N}$; in this economy, however, limited arbitrage is satisfied so that a competitive equilibrium exists. The initial allocation and a price vector assigning value zero to the second good defines such an equilibrium.

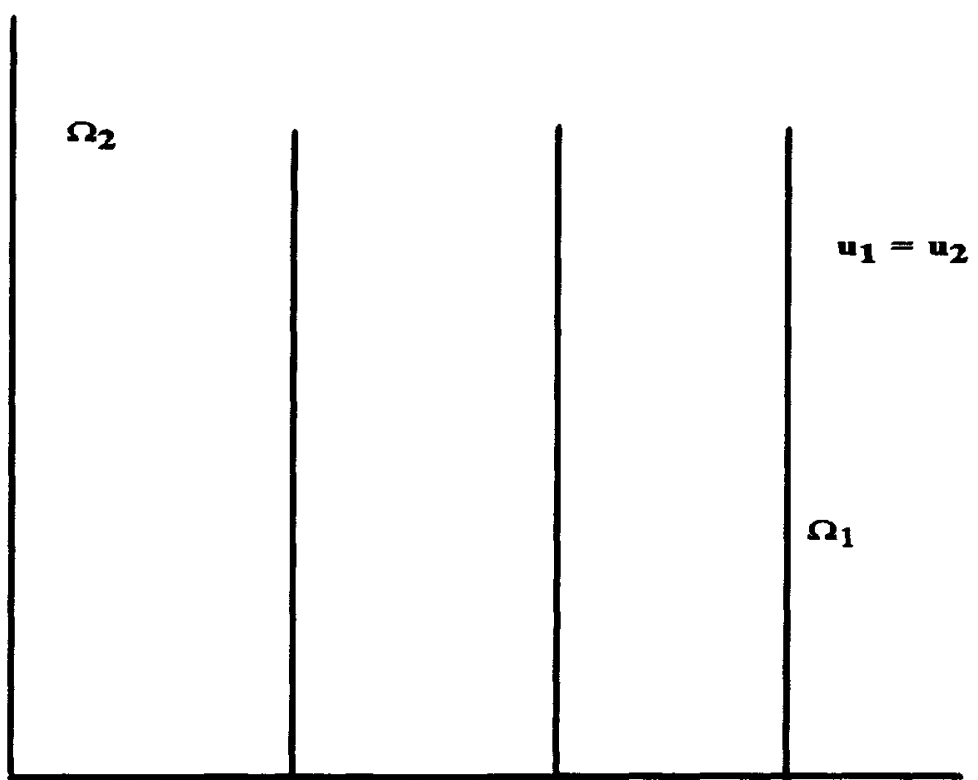


Figure 8. Equilibrium exists even when one trader has zero income

\section{Limited Arbitrage and the Compactness of the Pareto Frontier}

The Pareto frontier $P(\mathrm{E})$ is the set of feasible, efficient and individually rational utility allocations. With $H$ traders it is a subset of $R_{+}^{H}$. Proving the boundedness and closedness of the Pareto frontier is a crucial step in establishing the existence of a competitive equilibrium and the non-emptiness of the core. The main theorem of this section shows that limited arbitrage is necessary and sufficient for this.

There is a novel feature of the results which are presented here, a feature which is shared which those that were previously established in Chichilnisky (1991, 1992, $1994,1995,1995 \mathrm{a}, 1996 \mathrm{a})$ and Chichilnisky and Heal $(1992,1996)$. It starts from the observation that the compactness of the Pareto frontier need not imply the compactness of the set of feasible commodity allocations. The Pareto frontier is defined in utility space, $R_{+}^{H}$ while the commodity allocations are in the product of the commodity space with itself, $X^{H}$. When $X=R^{N}$, the commodity allocations are in $R^{H \times N}$. This observation is useful to distinguish the results presented here, in Chichilnisky $(1991,1992,1994,1995,1995 \mathrm{a}, 1996 \mathrm{a})$ and Chichilnisky and Heal $(1992,1996)$ from others in the literature. Other conditions used in the literature which are sufficient for the existence of an equilibrium and the core ensure that--with or without short sales-the set of individually rational and feasible commodity allocations is compact, see e.g. Chichilnisky and Heal (1993) Werner (1987) and Koutsougeras (1993) among others; the latter proves in detail that Werner's 1987 no-arbitrage condition, based on recession cones, implies the compactness of the set of feasible and individually rational allocations unless preferences are linear. But as already observed, and as is shown below, the boundedness of the set of feasible commodity allocations is not needed for existence. Indeed, such boundedness is not used in this paper, nor was it used in the results of Chichilnisky $(1991,1992,1994,1994 \mathrm{a}, 1995,1995,1996 \mathrm{a})$ and Chichilnisky and Heal $(1992,1996)$ : these are the first results in the literature proving the existence of equilibrium and the non-emptiness of the core in economies where limited arbitrage holds and the set of feasible and individually rational allocations is generally unbounded. In addition, of course, these results establish conditions which are simultaneously necessary and sufficient for the existence of equilibrium and the core, another novel feature. As a result, here the set of all possible efficient allocations, the contract curve, and the set of possible equilibria and the set of all possible core allocations, may be unbounded sets. Next we review some examples to illustrate and better appreciate the nature of the problems that can arise. 


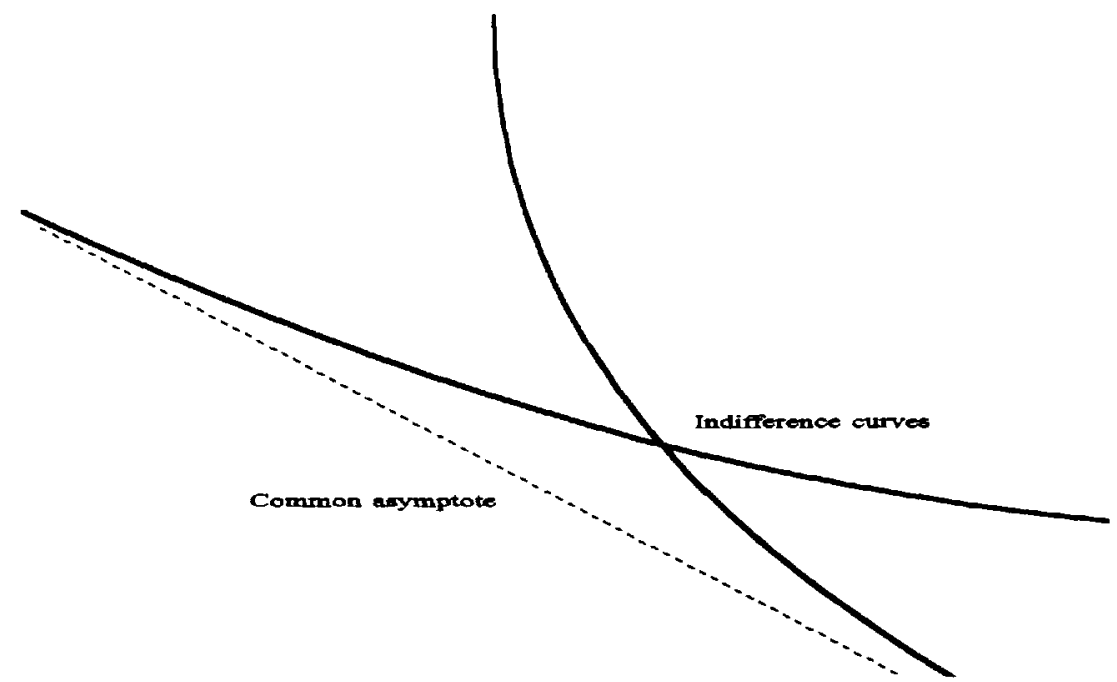

Figure 9. The Pareto frontier may fail to be closed even in finite dimensions

Example 5 Figure 9 shows that the Pareto frontier may fail to be closed even in finite dimensional models, provided the consumption set is the whole Euclidean space. It shows two traders with indifference curves having the line $y=-x$ as asymptote. Consumption sets are the whole space and feasible allocations are those which sum to zero. Utility functions are $u_{i}=x_{i}+y_{i} \pm e^{-\left(x_{i}-y_{i}\right)}, i=1,2$. Limited arbitrage rules out such cases.

Example 6 Another example is a two-agent economy where both agents have linear preferences: if the preferences are different the set of feasible utility allocations is unbounded. Of course, limited arbitrage rules out such situations.

Example 7 Even when the consumption set is bounded below, but the commodity space is infinite dimensional, examples can be provided where the Pareto frontier is not closed. ${ }^{36}$

Theorem 1 Consider an economy $\mathrm{E}$ as defined in Section 1. Then limited arbitrage is necessary and sufficient for the compactness of the Pareto frontier. ${ }^{37}$

Proof. This result always holds when the consumption set is bounded below by some vector in the space, ${ }^{38}$ and in that case it is proved using standard arguments, see e.g. Arrow and Hahn (1971). Therefore in the following I concentrate in the case where $X$ is unbounded.

Sufficiency first. Recall that by definition $P(\mathrm{E}) \subset U(\mathrm{E}) \subset R_{+}^{N}$. Proposition 4 and Corollary 1 proved that $U(E)$ is bounded when limited arbitrage is satisfied, so that $P(E)$ is bounded also.

The next step is to prove that $P(\mathrm{E})$ is closed when limited arbitrage is satisfied. Consider a sequence of allocations $\left\{z_{h}^{j}\right\}_{j=1,2 \ldots}, h=1,2, \ldots, H$, satisfying $\forall j, \sum_{h=1}^{H} z_{h}^{j} \leq$ $\Omega$, and $\lim _{j \rightarrow \infty} \sum_{h=1}^{H} z_{h}^{j}=\Omega$. Assume that $\left(u_{1}\left(z_{1}^{j}\right), \ldots, u_{H}\left(z_{H}^{j}\right)\right) \subset R_{+}^{N}$ converges to a 
utility allocation $v=\left(v_{1}, \ldots, v_{H}\right) \in R_{+}^{H}$, which is undominated by the utility allocation of any other feasible allocation. Observe that the vector $v$ may or not be the utility vector of a feasible allocation: when limited arbitrage is satisfied. I will prove that it is. The result is immediate if the set of feasible allocations is bounded; therefore I concentrate in the case where the set of feasible allocations is not bounded.

Let $M$ be the set of all traders $h \in\{1,2 .,,, H\}$, for whom the corresponding sequence of allocations $\left\{z_{h}^{j}\right\}_{j=1,2 \ldots}$ is bounded, i.e. $h \in M \Leftrightarrow \exists K_{h}:\left\|z_{h}^{j}\right\|<K_{h}<\infty$; let $J$ be its complement, $J=\{1,2, \ldots, H\}-M$, which I assume to be non empty. There exists a subsequence of the original sequence of allocations, which for simplicity is denoted also $\left\{z_{h}^{j}\right\}_{j=1,2 \ldots}, h=1,2, \ldots, H$, along which $\forall h \in M$, the $\lim _{j}\left\{z_{h}^{j}\right\}_{j=1,2 \ldots}=z_{h}$ exists, and $\sum_{h \in M} z_{h}+\lim _{j \rightarrow \infty} \sum_{h \in J} z_{h}^{j}=\Omega$. Recall that by Proposition 2 the cones $G_{h}$ are uniform, so that we may translate the origin of the space without loss of generality. Therefore we may assume without loss that $\sum_{h \in M} z_{h}=\Omega$, i.e. that $\lim _{j \rightarrow \infty} \sum_{h \in J} z_{h}^{j}=0$. For each $h \in J$, consider the normalized sequence $\left\{\frac{z_{h}^{j}}{\left\|z_{h}^{j}\right\|}\right\}_{j=1,2 \ldots}$, which is contained in a compact space, the unit ball. A convergent subsequence of this always exists, and is denoted also $\left\{\frac{z_{h}^{j}}{\left\|z_{h}^{j}\right\|}\right\}_{j=1,2 \ldots}$. Let $z_{h}=\lim _{j}\left\{\frac{z_{h}^{j}}{\left\|z_{h}^{j}\right\|}\right\}$. We showed in Proposition 4 that, under the conditions, $\forall h \in J, z_{h} \in \overline{G_{h}}$. If $\forall h \in J, z_{h} \notin G_{h}$ then by Proposition 2 eventually the utility values of the traders attain their limit for all $h$. the utility vector $v$ is achieved by a feasible allocation and the proof is complete. It remains therefore to consider only the case where for some trader $g \in J, z_{g} \in G_{g}$.

Define now the convex cone $C$ of all strictly positive linear combinations of the vectors $\left\{z_{h}\right\}_{h \in J}, C=\left\{w=\sum_{h \in J} \mu_{h} z_{h}, \mu_{h}>0\right\}$. There are two mutually exclusive and exhaustive cases: either (a) the cone $C$ is contained strictly in a half-space of $R^{N}$, or (b) the cone $C$ is a subspace of $R^{N}$. By construction $\lim _{j \rightarrow \infty} \sum_{h \in J} z_{h}^{j}=0$, which eliminates case (a). Therefore (b) must hold, and $C$ is a subspace of $R^{N}$. In particular, $-z_{g} \in C$, i.e. $\forall h \in J \exists \lambda_{h} \geq 0$ such that

$$
-z_{g}=\sum_{h \in J} \lambda_{h} z_{h}
$$

The final step is to show that (8) contradicts limited arbitrage. By limited arbitrage $\exists p \in \cap_{h} D_{h}$ s.t. $\left\langle p, z_{g}\right\rangle>0$, because $z_{g} \in G_{g}$, and $\forall h \in J,\left\langle p, z_{h}\right\rangle \geq 0$, since $z_{h} \in \bar{G}_{h}$. Therefore $\left\langle p, \sum_{h \in J} z_{h}\right\rangle \geq 0$, which contradicts (8). Since the contradiction arises from assuming that the Pareto frontier $P(\mathrm{E})$ is not closed, $P(\mathrm{E})$ must be closed. Therefore limited arbitrage implies a compact Pareto frontier.

Necessity is established next. If limited arbitrage fails, there is no vector $y \in H$ such that $\left\langle y, z_{h}\right\rangle>0$ for all $\left\{z_{h}\right\} \in G_{h}$. Equivalently, there exist a set $J$ consisting of at least two traders and, for each $h \in J$, a vector $z_{h} \in G_{h}$ such that $\sum_{h \in J} z_{h}=0$. Then by Proposition 2 either for some $h, z_{h} \in A_{h}$ so that the Pareto frontier is unbounded and therefore not compact, or else for some $h, z_{h} \in \partial G_{h} \cap G_{h}$ and therefore the Pareto frontier is not closed, and therefore not compact either. In either case, the Pareto frontier is not compact when limited arbitrage fails. Therefore compactness is necessary for limited arbitrage. 
Proposition 5 When $X=R^{N}$, limited arbitrage implies that the Pareto frontier $P(\mathrm{E})$ is homeomorphic to a simplex. ${ }^{39}$

Proof. This follows from Theorem 1 and by the convexity of preferences, cf. Arrow and Hahn (1971).

\section{Competitive Equilibrium and Limited Arbitrage}

This section establishes the main result linking the existence of a competitive equilibrium with the condition of limited arbitrage. ${ }^{40}$ The result is that limited arbitrage is simultaneously necessary and sufficient for the existence of a competitive equilibrium, ${ }^{41}$ and it was established first in Chichilnisky (1991, 1992, 1994a, 1995, 1996a). Other noteworthy features are: the equivalence between limited arbitrage and equilibrium applies equally to economies with or without short sales, and with or without strictly convex preferences. It therefore includes the Arrow Debreu market which has no short sales, a classic case which was neglected previously in the literature on no-arbitrage conditions. In addition, the equivalence applies to economies where the set of feasible and individually rational allocations may be unbounded, a case which has also been neglected in the literature. ${ }^{42}$ Finally, the equivalence between limited arbitrage and equilibrium extends to economies with infinitely many markets, see Chichilnisky and Heal $(1992,1993)$ and the next Section.

The result presented below was established first in Chichilnisky (1991, 1992, 1994a, 1995) for uniformly non-satiated convex preferences which are either all in case (i) e.g. strictly convex, or in case (ii), e.g. they have indifference surfaces with a closed set of gradient directions. The result presented here extends these earlier results in that it deals in a unified way with non-satiated convex preferences; in the same economy there may be a mixture of preferences of type (i) and (ii), see also Chichilnisky (1995a, 1996a):

Theorem 2 Consider an economy $\mathrm{E}=\left\{X, u_{h}, \Omega_{h}, h=1, \ldots, H\right\}$, where $H \geq 2$, with $X=R^{N}$ or $X=R_{+}^{N}$ and $N \geq 1$. Then the following two properties are equivalent:

(i) The economy $\mathrm{E}$ has limited arbitrage

(ii) The economy $\mathrm{E}$ has a competitive equilibrium

Proof. Necessity first. Consider first the case $X=R^{N}$ and assume without loss of generality that $\Omega_{h}=0$ for all $h$. The proof is by contradiction. Let $p^{*}$ be an equilibrium price and let $x^{*}=\left(x_{1}^{*}, \ldots x_{H}^{*}\right)$ be the corresponding equilibrium allocation. Then if limited arbitrage does not hold, $\exists h$ and $v \in G_{h}$ such that $\left\langle p^{*}, v\right\rangle \leq 0$, so that $\forall \lambda>0, \lambda v$ is affordable at prices $p^{*}$. However, $G_{h}$ is the same at any endowment by Proposition 2. It follows that $\exists \lambda>0: u_{h}\left(x_{h}^{*}+\lambda v\right)>u_{h}\left(x_{h}^{*}\right)$, which contradicts the fact that $x_{h}^{*}$ is an equilibrium allocation. This completes the proof of necessity when $X=R^{N}$.

Consider next $X=R_{+}^{N}$. Assume that $\forall q \in S(\mathrm{E}) \exists h \in\{1, \ldots, H\}$ such that $\left\langle q, \Omega_{h}\right\rangle=0$. Then if limited arbitrage is not satisfied $\cap_{h=1}^{H} D_{h}^{+}\left(\Omega_{h}\right)=\emptyset$, which implies 
that $\forall p \in R^{N}$. $\exists h$ and $v(p) \in G_{h}\left(\Omega_{h}\right)$ :

$$
\langle p, \lambda v(p)\rangle \leq 0, \forall \lambda>0
$$

I will now show that this implies that a competitive equilibrium price cannot exist. By contradiction. Let $p^{*}$ be an equilibrium price and $x^{*} \in X^{H}$ be the corresponding equilibrium allocation. Consider $v\left(p^{*}\right) \in G_{h}\left(\Omega_{h}\right)$ satisfying (9). If $\lim _{\lambda \rightarrow \infty} u_{h}\left(\Omega_{h}+\right.$ $\lambda v(p))=\infty$ this leads directly to a contradiction, because $\left\langle p^{*}, \lambda v\left(p^{*}\right)\right\rangle \leq 0$, so that for all $\lambda . \lambda v\left(p^{*}\right)$ is affordable, and therefore there is no affordable allocation which maximizes $h^{\prime} s$ utility at the equilibrium price $p^{*}$. Consider next the case where $v\left(p^{*}\right) \in G_{h}\left(\Omega_{h}\right)-A_{h}\left(\Omega_{h}\right)$. By definition, $u_{h}\left(\Omega_{h}+\lambda v\left(p^{*}\right)\right)$ never ceases to increase in $\lambda$, and $\lim _{\lambda \rightarrow \infty} u_{h}\left(\Omega_{h}+\lambda v\left(p^{*}\right)\right)<\infty$. If $u_{h}\left(x_{h}^{*}\right)>\lim _{\lambda \rightarrow \infty} u_{h}\left(\Omega_{h}+\lambda v\left(p^{*}\right)\right.$ then there exists a vector, namely $x_{h}^{*}$, which has utility strictly larger than $v\left(p^{*}\right) \in \partial G_{h}\left(\Omega_{h}\right)$ so that, as shown in Proposition 2, the direction defined by the vector $x^{*}-\Omega_{h}$ must be contained in $A_{h}\left(\Omega_{h}\right)$. But this contradicts the assumption that $x_{h}^{*}$ is an equilibrium allocation, because if $x^{*}-\Omega_{h} \in A_{h}\left(\Omega_{h}\right), \lim _{\lambda \rightarrow \infty} u_{h}\left(\Omega_{h}+\lambda\left(x_{h}^{*}-\Omega_{h}\right)\right)=\infty$, while $\left\langle p^{*}, \lambda\left(x_{h}^{*}-\Omega_{h}\right)\right\rangle \leq 0$ so that $x_{h}^{*}$ cannot be an equilibrium allocation. Therefore limited arbitrage is also necessary for the existence of a competitive equilibrium in this case.

It remains to consider the case where $\exists p \in S(\mathrm{E})$ such that $\forall h \in\{1, \ldots, H\},\left\langle p, \Omega_{h}\right\rangle \neq$ 0 . But in this case by definition $\bigcap_{h=1}^{H} D_{h}^{+}\left(\Omega_{h}\right) \neq \emptyset$ since $\forall h \in\{1 \ldots H\}, R_{++}^{N} \subset$ $D_{h}^{+}\left(\Omega_{h}\right)$, so that limited arbitrage is always satisfied when an equilibrium exists. This completes the proof of necessity.

Sufficiency next. The proof uses the fact that the Pareto frontier is homeomorphic to a simplex. When $X=R_{+}^{N}$ the Pareto frontier of the economy $P(\mathrm{E})$ is always homeomorphic to a simplex, see Arrow and Hahn (1971). In the case $X=R^{N}$ this may fail. However, by Theorem 1 above, if the economy satisfies limited arbitrage then the Pareto frontier is compact; under the assumptions on preferences, it is then also homeomorphic to a simplex (Arrow and Hahn (1971)). Therefore in both cases, $P(\mathrm{E})$ is homeomorphic to a simplex and one can apply the by now standard Negishi method of using a fixed point argument on the Pareto frontier to establish the existence of a pseudoequilibrium. ${ }^{43}$ It remains however to prove that the pseudoequilibrium is also a competitive equilibrium.

To complete the proof of existence of a competitive equilibrium consider first $X=R^{N}$. Then $\forall h=1, \ldots, H$ there exists an allocation in $X$ of strictly lower value than the pseudoequilibrium $x_{h}^{*}$ at the price $p^{*}$. Therefore by Lemma 3, Chapter 4 , page 81 of Arrow and Hahn (1971), the quasi-equilibrium $\left(p^{*}, x^{*}\right)$ is also a competitive equilibrium, completing the proof of existence when $X=R^{N}$.

Next consider $X=R_{+}^{N}$, and a quasi-equilibrium $\left(p^{*}, x^{*}\right)$ whose existence was already established. If every individual has a positive income at $p^{*}$, i.e. $\forall h,\left\langle p^{*}, \Omega_{h}\right\rangle>$ 0 , then by Lemma 3, Chapter 4 of Arrow and Hahn (1971) the quasi-equilibrium $\left(p^{*}, x^{*}\right)$ is also a competitive equilibrium, completing the proof. Furthermore, observe that in any case the pseudoequilibrium price $p^{*} \in S(\mathrm{E})$, so that $S(\mathrm{E})$ is not empty. To prove existence we consider therefore two cases: first the case where $\exists q^{*} \in S(\mathrm{E}): \forall h$, $\left\langle q^{*}, \Omega_{h}\right\rangle>0$. In this case, by the above remarks from Arrow and Hahn $(1971),\left(q^{*}, x^{*}\right)$ 
is a competitive equilibrium. The second case is when $\forall q \in S(\mathrm{E}), \exists h \in\{1, \ldots, H\}$ such that $\left\langle q . \Omega_{h}\right\rangle=0$. Limited arbitrage then implies:

$$
\exists q^{*} \in S(\mathrm{E}): \forall h,\left\langle q^{*}, v\right\rangle>0 \text { for all } v \in G_{h}\left(\Omega_{h}\right) .
$$

Let $x^{*}=x_{1}^{*} \ldots, x_{H}^{*} \in X^{H}$ be a feasible allocation in $\Upsilon$ supported by the vector $q^{*}$ defined in (10): by definition, $\forall h, u_{h}\left(x_{h}^{*}\right) \geq u_{h}\left(\Omega_{h}\right)$ and $q^{*}$ supports $x^{*}$. Note that any $h$ minimizes costs at $x_{h}^{*}$ because $q^{*}$ is a support. Furthermore $x_{h}^{*}$ is affordable under $q^{*}$. Therefore, $\left(q^{*}, x^{*}\right)$ can fail to be a competitive equilibrium only when for some $h,\left\langle q^{*}, x_{h}^{*}\right\rangle=0$, for otherwise the cost minimizing allocation is always also utility maximizing in the budget set $B_{h}\left(q^{*}\right)=\left\{w \in X:\left\langle q^{*}, w\right\rangle=\left\langle q^{*}, \Omega_{h}\right\rangle\right\}$.

It remains therefore to prove existence when $\left\langle q^{*}, x_{h}^{*}\right\rangle=0$ for some $h$. Since by the definition of $S(\mathrm{E}), x^{*}$ is individually rational, i.e. $\forall h, u_{h}\left(x_{h}^{*}\right) \geq u_{h}\left(\Omega_{h}\right)$, then $\left\langle q^{*}, x_{h}^{*}\right\rangle=0$ implies $\left\langle q^{*}, \Omega_{h}\right\rangle=0$, because by definition $q^{*}$ is a supporting price for the equilibrium allocation $x^{*}$. If $\forall h, u_{h}\left(x_{h}^{*}\right)=0$ then $x_{h}^{*} \in \partial R_{+}^{N}$, and by the monotonicity and quasi-concavity of $u_{h}$, any vector $y$ in the budget set defined by the price $p^{*}, B_{h}\left(q^{*}\right)$, must also satisfy $u_{h}(y)=0$, so that $x_{h}^{*}$ maximizes utility in $B_{h}\left(q^{*}\right)$, which implies that $\left(q^{*}, x^{*}\right)$ is a competitive equilibrium. Therefore $\left(q^{*}, x^{*}\right)$ is a competitive equilibrium unless for some $h, u_{h}\left(x_{h}^{*}\right)>0$.

Assume therefore that the quasiequilibrium $\left(q^{*}, x^{*}\right)$ is not a competitive equilibrium, and that for some $h$ with $\left\langle q^{*}, \Omega_{h}\right\rangle=0, u_{h}\left(x_{h}^{*}\right)>0$. Since $u_{h}\left(x_{h}^{*}\right)>0$ and $x_{h}^{*}$ $\in \partial R_{+}^{N}$ then an indifference surface of a commodity bundle of positive utility $u_{h}\left(x_{h}^{*}\right)$ intersects $\partial R_{+}^{N}$ at $x_{h}^{*} \in \partial R_{+}^{N}$. Let $r$ be the ray in $\partial R_{+}^{N}$ containing $x_{h}^{*}$. If $w \in r$ then $\left\langle q^{*}, w\right\rangle=0$, because $\left\langle q^{*}, x_{h}^{*}\right\rangle=0$. Since $u_{h}\left(x_{h}^{*}\right)>0$, by Proposition $3 u_{h}$ strictly increases along $r$, so that $w \in G_{h}\left(x_{h}^{*}\right)$. But this contradicts the choice of $q^{*}$ as a supporting price satisfying limited arbitrage (10) since

$$
\exists h \text { and } w \in G_{h}\left(\Omega_{h}\right) \text { such that }\left\langle q^{*}, w\right\rangle=0 .
$$

The contradiction between (11) and (10) arose from the assumption that $\left(q^{*}, x^{*}\right)$ is not a competitive equilibrium, so that $\left(q^{*}, x^{*}\right)$ must be a competitive equilibrium, and the proof is complete.

\section{Economies with Infinitely Many Markets}

The results of Theorem 2 are also valid for infinitely many markets. As already seen, the existence of inner products is useful in defining limited arbitrage. For this reason and because of the natural structure of prices in Hilbert spaces, I work on a Hilbert space of commodities in which inner products are defined.

\subsection{Hilbert Spaces and the Cone Condition}

All Hilbert spaces have positive orthants with empty interior. This can make things difficult when seeking to prove the existence of an equilibrium, which depends on finding supporting prices for efficient allocations. Supporting prices are usually found by 
applying the Hahn-Banach theorem, and without such prices a competitive equilibrium does not exist. Therefore the Hahn Banach theorem is crucial for proving existence of an equilibrium. However this theorem requires that the convex set being supported has a non-empty interior. a condition which is never satisfied within the positive orthant of a Hilbert space. This problem, which is typical in infinite dimensional spaces. was solved in 1980 by Chichilnisky and Kalman (1980) who introduced a condition on preferences, the cone condition (C-K.) and proved that it is necessary and sufficient for separating convex sets with or without non-empty interior. thus extending Hahn-Banach's theorem to encompass all convex sets, whether or not they have an empty interior. Since its introduction the $\mathbf{C}-\mathbf{K}$ cone condition has been used extensively to prove the existence of a market equilibrium and in game theory; it is now a standard condition of economies with infinitely many markets and is known also under the name of "properness", cf. Chichilnisky (1993a).

In addition to the cone condition, one more result is needed to extend directly the proof of Theorem 2 to economies with infinitely many markets: the compactness of the Pareto frontier. Recall that this frontier is always a finite dimensional object when there are a finite number of traders: it is contained in $R_{+}^{H}$, where $H$ is the number of traders.

\subsection{Limited Arbitrage and the Cone Condition}

A somewhat unexpected result is that limited arbitrage implies the $\mathbf{C - K}$ cone condition, see Chichilnisky and Heal $(1992,1995)$. Because of this, limited arbitrage is necessary and sufficient for the existence of a competitive equilibrium and the core, with or without short sales, in the infinite dimensional space $\mathrm{H}$. Limited arbitrage therefore unifies the treatment of finitely and infinitely many markets.

Consider an economy $\mathrm{E}$ as defined in Section 2 except that here $X=\mathrm{H}$ or $X=\mathrm{H}^{+}$; more general convex sets can be considered as well, see (Chichilnisky and Heal 1992, 1995). The global cones and the market cones, and the limited arbitrage condition, are the same as defined in the finite dimensional cases when $X=R^{N}$ and $X=R_{+}^{N}$ respectively. To shorten the presentation, here the market cones are assumed to be uniform across initial endowments, a condition which is automatically satisfied under Assumption 1 when $X=\mathrm{H}$, and which is not needed for the main results, cf. Chichilnisky and Heal $(1992,1995)$. Therefore here either limited is satisfied at every endowment or not at all. The results on existence of an equilibrium presented below are due to Chichilnisky and Heal $(1992,1995)$.

Definition 9 The cone defined by a convex set $D \subset X$ at a point $x \in D$ is $C(D, x)=$ $\{z \in \mathrm{X}: z=x+\lambda(y-x)$, where $\lambda>0$ and $y \in D\}$.

Definition 10 A convex set $D \subset \mathrm{X}$ satisfies the $\mathbf{C - K}$ cone condition of (Chichilnisky and Kalman. 1980) at $x \in \mathrm{D}$ when there exists a vector $v \in \mathrm{X}$ which is at positive distance $\varepsilon(D, x)$ from the cone with vertex $x$ defined by the set $D, C(D, x)$. 
Definition $11 A$ preference $u_{h}: X \rightarrow R$ satisfies the $\mathbf{C - K}$ cone condition of Chichilnisky and Kalman $(1980)^{44}$ when for every $x \in \mathrm{X}$, the preferred set $u_{h}^{x}=\{y$ : $\left.u_{h}(y) \geq u_{h}(x)\right\} \subset X$ of $u_{h}$ at $x$ satisfies the $\mathrm{C}-\mathrm{K}$ condition, and $\varepsilon\left(P_{x}, x\right)$ is independent of $x$.

The finite dimensional proofs work for infinite dimensions when $X$ is a Hilbert space $H$. see Chichilnisky and Heal $(1992,1995)$. The only case which requires special treatment is $X=H^{+}$because with infinite dimensional Hilbert spaces the positive orthant $H^{+}$has empty interior:

Theorem 3 (Chichilnisky and Heal) Consider an economy $\mathrm{E}$ as defined in Section 2, where the trading space is either $X=\mathrm{H}^{+}$, or $X=\mathrm{H}$, and where $\mathrm{H}$ is a Hilbert space of finite or infinite dimensions. Then limited arbitrage implies the $\mathbf{C}-\mathbf{K}$ (Chichilnisky and Kalman 1980) cone condition. In particular, the second welfare theorem applies under limited arbitrage: a Pareto efficient allocation is also a competitive equilibrium.

Proof. For a proof see Chichilnisky and Heal $(1992,1995)$. An outline of the proof for $X=H^{+}$follows. The case $X=H$ is in Chichilnisky and Heal (1992 and 1995) and follows directly from the finite dimensional case.

Let $X=\mathrm{H}^{+}$: I will show first that limited arbitrage, as defined in Section 2, implies that there exists a vector $p \neq 0$ in $S(\mathrm{E})$. The proof is by contradiction. If $\sim$ $\exists p \neq 0$ in $S(\mathbb{E})$, then the intersection of the dual cones in Definition 6 must be empty, i.e. $\cap_{h=1}^{H} D_{h}^{+}=\emptyset$ : this occurs either because for some $h$, the set $D_{h}^{+}=D_{h} \cap S(\mathrm{E})$ is empty, or alternatively because the set $S(\mathrm{E})$ itself is empty. In either case this leads to a contradiction with limited arbitrage which requires that $\cap_{h=1}^{H} D_{h}^{+} \neq \emptyset$. Since the contradiction arises from assuming that $\sim \exists p \neq 0$ in $S(E)$, it follows that $\exists p \in S(\mathrm{E}), p \neq 0$, i.e. the preferred set of $u_{h}$ can be supported by a non-zero price $p$ at some $x_{h}$ which is part of a feasible affordable efficient and individually rational allocation, $x=x_{1}, \ldots, x_{H}$.

The last step is to show that there exists one vector $v$, the same for all traders, which is at a positive distance $\varepsilon$ from $C\left(u_{h}^{x}, x\right)$ for every trader $h$ as well as for every $x \in X$. Consider now the vector $v=\sum_{h=1}^{H} p_{h}$, where $p_{h}$ is the support whose existence was established above, and let $\varepsilon=\min _{i=1,2, \ldots, H}\left\{\varepsilon_{i}\right\}$. The vector $v$ satisfies the definition of the cone condition C-K. ${ }^{45}$

Theorem 4 (Chichilnisky and Heal) Consider an economy $\mathrm{E}$ as defined in Section 2, where $X=\mathrm{H}$, or $X=\mathrm{H}^{+}$, where $\mathrm{H}$ is a Hilbert space of finite or infinite dimensions. Then limited arbitrage is necessary and sufficient for the compactness of the Pareto frontier.

Proof. Since the cone condition holds, the proof is a straightforward extension of Theorem 1 which holds for the finite dimensional case. See Chichilnisky and Heal $(1992,1995)$. 
Theorem 5 (Chichilnisky and Heal) Consider an economy $\mathrm{E}$ as defined in Section 2, where $X=\mathrm{H}^{+}$or $X=\mathrm{H}$. a Hilbert space of finite or infinite dimensions. Then limited arbitrage is necessary and sufficient for the existence of a competitive equilibrium.

Proof. The proof is similar to that for the finite dimensional case, see Chichilnisky and Heal $(1992,1995)$.

\subsection{Subeconomies with Competitive Equilibria}

The condition of limited arbitrage need not be tested on all traders simultaneously: in the case of $R^{N}$, it needs only be satisfied on subeconomies with no more traders than the number of commodities in the economy, ${ }^{46} N$, plus one.

Definition $12 A k$-trader sub-economy of $\mathrm{E}$ is an economy $\mathrm{F}$ consisting of a subset of $k \leq H$ traders in $\mathrm{E}$, each with the endowments and preferences as in $\mathbf{E}: \mathbf{F}=$ $\left\{X, u_{h}, \Omega_{h}, h \in J \subset\{1, \ldots, H\}\right.$, cardinality $\left.(J)=k \leq H\right\}$.

Theorem 6 The following four properties of an economy $\mathrm{E}$ with trading space $R^{N}$ are equivalent:

(i) E has a competitive equilibrium

(ii) Every sub economy of $\mathrm{E}$ with at most $N+1$ traders has a competitive equilibrium

(iii) E has limited arbitrage

(iv) $\mathrm{E}$ has limited arbitrage for any subset of traders with no more that $N+1$ members.

Proof. Theorem 1 implies (i) $\Leftrightarrow$ (iii) and (ii) $\Leftrightarrow$ (iv). That (iii) $\Leftrightarrow$ (iv) follows from the following theorem which is a corollary in Chichilnisky (1993c): Consider a family $\left\{U_{i}\right\}_{i=1 \ldots H}$ of convex sets in $R^{N}, H, N \geq 1$. Then

$$
\bigcap_{i=1}^{H} U_{i} \neq \emptyset \text { if and only if } \bigcap_{j \in J} U_{i} \neq \emptyset
$$

for any subset of indices $J \subset\{1 \ldots H\}$ having at most $N+1$ elements.

In particular, an economy $\mathrm{E}$ as defined in Section 2 satisfies limited arbitrage, if and only if it satisfies limited arbitrage for any subset of $k=N+1$ traders, where $N$ is the number of commodities in the economy $E$.

\section{Limited Arbitrage Equilibrium and The Core with Fi- nitely or Infinitely Many Markets}

Limited arbitrage is also necessary and sufficient for the nonemptiness of the core: ${ }^{44}$ 
Theorem 7 Consider an economy $\mathbf{E}=\left\{X, u_{h}, \Omega_{h}, h=1, \ldots . H\right\}$, where $H \geq 2$, $X=R^{N}$ and $N \geq 1$, or $X$ is a Hilbert space $H$. Then the following three properties are equivalent:

(i) The economy $\mathbf{E}$ has limited arbitrage

(ii) The economy $\mathrm{E}$ has a core

(iii) Every subeconomy of $E$ with at most $N+1$ trades has a core

Proof. For the proof of (i) $\Leftrightarrow$ (ii) and a discussion of the literature see Chichilnisky (1996). ${ }^{45}$

The equivalence (i) $\Leftrightarrow$ (iii) then follows from Theorem 6 .

\section{Social Diversity and the Supercore}

The supercore was defined and motivated in Section 1.2. It measures the extent to which a society has reasons to stay together. Social diversity comes in many shades, one of which, the mildest possible, will be used to establish the existence of a supercore:

Definition 13 An economy $\mathrm{E}$ is socially diverse when it does not satisfy limited arbitrage. When $X=R^{N}$, this means:

$$
\bigcap_{h=1}^{H} D_{h}=\emptyset .
$$

When $X=R_{+}^{N}$ :

$$
\bigcap_{h=1}^{H} D_{h}^{+}\left(\Omega_{h}\right)=\emptyset .
$$

In this section short sales are allowed, so that the trading space is $X=R^{N}$. To simplify notation I assume without loss of generality that all endowments are zero, $\forall h, \Omega_{h}=0$. Assume now that the normalized gradients of closed sets of indifferent vectors define closed sets (case (ii)) so that ${ }^{48} G_{h}=A_{h}$. 
Definition $14 \mathrm{E}$ has social diversity of type 1, or SD1. when all sub economies with at most $H-1$ traders have limited arbitrage, but $\mathrm{E}$ does not.

Theorem 8 Consider an economy $\mathrm{E}$ with at least three traders. Then if $\mathrm{E}$ has social diversity of type 1. SD1. its supercore is not empty.

Proof. To simplify notation, assume without loss of generality that $\forall h, \Omega_{h}=0$. Since the economy has social diversity of type 1, every subeconomy of $H-1$ traders satisfies limited arbitrage, which by Proposition 4 implies that gains from trade $G(E)$ are bounded in every $H-1$ trader subeconomy. In particular, there is a maximum level of utility which each trader can obtain by him or herself, and the same is true for any subgroup consisting of at most $H-1$ traders.

However, by Proposition 4, gains from trade cannot be bounded in $E$ for the set of all $H$ traders, since $\mathbf{E}$ does not satisfy limited arbitrage.

\section{Limited Arbitrage and Social Choice}

Limited arbitrage is also crucial for achieving resource allocation via social choice. Two main approaches to social choice are studied here. One is Arrow's: his axioms of social choice require that the social choice rule $\Phi$ be non-dictatorial, independent of irrelevant alternatives, and satisfy a Pareto condition (Arrow (1951)). A second approach requires, instead, that the rule $\Phi$ be continuous, anonymous, and respect unanimity, Chichilnisky (1980 and 1982). Both approaches have led to corresponding impossibility results (Arrow 1951, Chichilnisky $(1980,1982)$ ). Though the two sets of axioms are quite different, it has been shown recently that the impossibility results which emerge from them are equivalent, see Baryshnikov (1993). Furthermore, as is shown below, limited arbitrage is closely connected with both sets of axioms. Economies which satisfy limited arbitrage admit social choice rules with either set of axioms. Therefore, in a well defined sense, the social choice problem can only be solved in those economies which satisfy limited arbitrage.

How do we allocate resources by social choice? Social choice rules assign a social preference $\Phi\left(u_{1} \ldots u_{H}\right)$ to each list $\left(u_{1} \ldots u_{H}\right)$ of individual preferences of an economy E. ${ }^{46}$ The social preference ranks allocations in $R^{N \times H}$, and allows to select an optimal feasible allocation. This is the resource allocation obtained via social choice.

The procedure requires, of course, that a social choice rule $\Phi$ exists: the role of limited arbitrage is important because it ensures existence. This will be established below. I prove here that limited arbitrage is necessary and sufficient for resolving Arrow's paradox when the domain of individual preferences are those in the economy, and the choices are those feasible allocations which give large utility value. ${ }^{47}$

Limited arbitrage provides a restriction on the relationship between individual preferences under which social choice rules exist. A brief background on the matter of preference diversity follows.

Arrow's impossibility theorem established that in general a social choice rule $\Phi$ does not exist: the problem of social choice has no solution unless individual prefer- 
ences are restricted. Duncan Black (1948) established that the "single peakedness" of preferences is a sufficient restriction to obtain majority rules. Using different axioms. Chichilnisky (1980 and 1982) established also that a social choice rule $\Phi$ does not generally exist; subsequently Chichilnisky and Heal (1983) established a necessary and sufficient restriction for the resolution of the social choice paradox: the contractibility of the space of preferences. ${ }^{48}$ Contractibility can be interpreted as a limitation on preference diversity, Heal (1983). In all cases, therefore, the problem of social choice is resolved by restricting the diversity of individual preferences. The main result in this section is that the restriction on individual preferences required to solve the problem is precisely limited arbitrage. The connection between limited arbitrage and contractibility is discussed below.

The section is organized as follows. First I show in Proposition 6 that the economy E satisfies limited arbitrage if and only if it contains no Condorcet cycles on choices of large utility values. ${ }^{49}$ Condorcet cycles are the building blocks of Arrow's impossibility theorem, and are at the root of the social choice problem. On the basis of Proposition 6 , I prove in Theorem 9 that limited arbitrage is necessary and sufficient for resolving Arrow's paradox on allocations of large utility values.

Definition 15 A Condorcet cycle is a collection of three preferences over a choice set $X$. represented by three utilities $u_{i}: X \rightarrow R, i=1,2,3$, and three choices $\alpha, \beta, \gamma$ within a feasible set $Y \subset X$ such that $u_{1}(\alpha)>u_{1}(\beta)>u_{1}(\gamma), u_{2}(\gamma)>u_{2}(\alpha)>u_{2}(\beta)$ and $u_{3}(\beta)>u_{3}(\gamma)>u_{3}(\alpha)$.

Within an economy with finite resources $\Omega>>$, the social choice problem is about the choice of allocations of these resources. Choices are in $X=R^{N \times H}$. An allocation $\left(x_{1} \ldots x_{H}\right) \in R^{N \times H}$ is feasible if $\sum_{i} x_{i}-\Omega=0$. Consider an economy $E$ as defined in Section 2. Preferences over private consumption are increasing, $u_{h}(x)>u_{h}(y)$ if $x>y \in R^{N}$, utilities are uniformly non-satiated (Assumption 1), and indifference surfaces which are not bounded below have a closed set of gradients, ${ }^{50}$ so that $G_{h}=A_{h}$. While the preferences in $\mathrm{E}$ are defined over private consumption, they naturally define preference over allocations, as follows: define $u_{h}\left(x_{1} \ldots x_{H}\right) \geq u_{h}\left(y_{i} \ldots y_{H}\right) \Leftrightarrow u_{h}\left(x_{h}\right) \geq u_{h}\left(y_{h}\right)$. Thus the preferences in the economy $E$ induce naturally preferences over the feasible allocations in $E$.

Definition 16 The family of preferences $\left\{u_{1} \ldots u_{H}\right\}, u_{h}: R^{N} \rightarrow R$ of an economy $\mathrm{E}$ has a Condorcet cycle of size $k$ if for every three preferences $u_{1}^{k}, u_{2}^{k}, u_{3}^{k} \in\left\{u_{1} \ldots u_{H}\right\}$ there exists three feasible allocations $\alpha^{k}=\left(\alpha_{1}^{k}, \alpha_{2}^{k}, \alpha_{3}^{k}\right) \in X^{3 \times H} \subset R^{3 \times N \times H} ; \beta^{k}=$ $\left(\beta_{1}^{k}, \beta_{2}^{k}, \beta_{3}^{k}\right)$ and $\gamma^{k}=\left(\gamma_{1}^{k}, \gamma_{2}^{k}, \gamma_{3}^{k}\right)$ which define a Condoncet cycle, and such that each trader $h=1, \ldots, H$, achieves at least a utility level $k$ at each choice:

$$
\min _{h=1, \ldots, H}\left\{\left[u_{h}^{k}\left(\alpha_{h}^{k}\right), u_{h}^{k}\left(\beta_{h}^{k}\right), u_{h}\left(\gamma_{h}^{k}\right)\right]\right\}>k .
$$

The following shows that limited arbitrage eliminates Condorcet cycles on matters of great importance, namely on those with utility level approaching the supremum 
of the utilities, which for simplicity and without loss of generality we have assumed to be $\infty$ :

Proposition 6 Let $\mathrm{E}$ be a market economy with short sales $\left(X=R^{N}\right)$ and $H \geq$ 3 traders. Then $\mathrm{E}$ has social diversity if and only if its traders' preferences have Condorcet cycles of every size. Equivalently, $\mathrm{E}$ has limited arbitrage if and only for some $k>0$, the traders ' preferences have no Condorcet cycles of size larger than $k$.

Proof. Consider an economy with Condorcet cycles of all sizes. For each $k>0$, there exists three allocations denoted $\left(\alpha^{k}, \beta^{k}, \gamma^{k}\right) \in R^{3 \times N \times H}$ and three traders $u_{k_{1}}^{k}, u_{k_{2}}^{k}, u_{k_{3}}^{k} \subset\left\{u_{1} \ldots u_{H}\right\}$ which define a Condorcet triple of size $k$. By definition, for every $k$, each of the three allocations is feasible, for example, $\alpha^{k}=\left(\alpha_{1}^{k}, \ldots, \alpha_{H}^{k}\right) \in$ $R^{N \times H}$. and $\sum_{i=1}^{H}\left(\alpha_{i}^{k}\right)=0$. Furthermore $\min _{h=1, \ldots, H}\left\{\left[u_{h}^{k}\left(\alpha_{h}^{k}\right), u_{h}^{k}\left(\beta_{h}^{k}\right), u_{h}\left(\gamma_{h}^{k}\right)\right]\right\}>k$, so that e.g. $\lim _{k \rightarrow \infty}\left(u_{h}\left(\alpha_{h}^{k}\right)\right)=\infty$. There exist therefore a sequence of allocations $\left(\theta^{k}\right)_{k=1,2 \ldots}=\left(\theta_{1}^{k}, \ldots, \theta_{H}^{k}\right)_{k=1,2 \ldots}$ such that $\forall k, \sum_{h=1}^{H} \theta_{h}^{k}=0$ and $\forall h \sup _{k \rightarrow \infty}\left(\inf _{i=1,2,3}\left(u_{h}\left(\theta_{h}^{k}\right)\right)=\right.$ $\infty$. This implies that $E$ has unbounded gains from trade, which contradicts Proposition 3. Therefore $E$ cannot have Condorcet cycles of every size.

Conversely, if $E$ has no limited arbitrage, for any $k>0$, there exist a feasible allocation $\left(a_{1}^{k}, a_{2}^{k}, \ldots, a_{H}^{k}\right)$, such that $\sum_{h=1}^{H} a_{h}^{k} \leq 0$, and $\forall h, u_{h}\left(a_{h}^{k}\right) \geq k$. For each integer $k>0$, and for a small enough $\varepsilon>0$ define now the vector $\Delta=(\varepsilon, \ldots, \varepsilon) \in R_{+}^{N}$ and the following three allocations: $\alpha^{k}=\left(k a_{1}^{k}, k a_{2}^{k}-2 \Delta, k a_{3}^{k}+2 \Delta, k a_{4}^{k}, \ldots, k a_{H}^{k}\right), \beta^{k}=$ $\left(k a_{1}^{k}-\Delta, k a_{2}^{k}, k a_{3}^{k}+\Delta, k a_{4}^{k}, \ldots, k a_{H}^{k}\right)$ and $\gamma^{k}=\left(k a_{1}^{k}-2 \Delta, k a_{2}^{k}-\Delta, k a_{3}^{k}+3 \Delta, k a_{4}^{k}, . . k a_{H}^{k}\right)$. Each allocation is feasible, e.g. $k a_{1}^{k}+k a_{2}^{k}-2 \Delta+k a_{3}^{k}+2 \Delta+k a_{4}^{k}+\ldots+k a_{H}^{k}=$ $k\left(\sum_{h=1}^{H} a_{h}^{k}\right) \leq 0$. Furthermore for each $k>0$ sufficiently large, the three allocations $\alpha^{k}, \beta^{k}, \gamma^{k}$ and the traders $h=1,2,3$, define a Condorcet cycle of size $k$ : all traders except for $1,2,3$, are indifferent between the three allocations and they reach a utility value at least $k$, while trader 1 prefers $\alpha^{k}$ to $\beta^{k}$ to $\gamma^{k}$, trader 3 prefers $\gamma^{k}$ to $\alpha^{k}$ to $\beta^{k}$, and trader 2 prefers $\beta^{k}$ to $\gamma^{k}$ to $\alpha^{k}$. Observe that this construction can be made for any three traders within the set $\{1,2, \ldots, H\}$. This completes the proof.

The next result uses Proposition 6 to establish the connection between limited arbitrage and Arrow's theorem. Consider Arrow's three axioms: Pareto, independence of irrelevant alternatives, and non-dictatorship. The social choice problem is to find a social choice rule $\Phi: P^{j} \rightarrow P$ from individual to social preferences satisfying Arrow's three axioms; the domain for the rule $\Phi$ are profiles of individual preferences over allocations of the economy E: $\Phi: P^{j} \rightarrow P$. Recall that each preference in the economy $E$ defines a preference over feasible allocations in $E$.

Definition 17 The economy $\mathrm{E}$ admits a resolution of Arrow's paradox if for any number of voters $j \geq 3$ there exists a social choice function from the space $P=$ $\left\{u_{1}, \ldots, u_{H}\right\}$ of preferences of the economy $\mathbf{E}$ into the space $Q$ of complete transitive preference defined on the space of feasible allocations of $\mathrm{E}, \Phi: P^{j} \rightarrow Q$, satisfying Arrow's three axioms. 
Definition 18 A feasible allocation $\left(\alpha_{1}^{k}, \ldots, \alpha_{H}^{k}\right) \in R^{N \times H}$ has utility value $k$, or simply value $k$, if each trader achieves at least a utility level $k$ :

$$
\min _{h \in H}\left\{\left[u_{1}^{k}\left(\alpha_{1}^{k}\right) \ldots, u_{H}\left(\alpha_{H}^{k}\right)\right]\right\}>k
$$

Definition 19 Arrow's paradox is said to be resolved on choices of large utility value in the economy $\mathrm{E}$ when for all $j \geq 3$ there exists social choice function $\Phi: P^{j} \rightarrow Q$ and $a k>0$ such that $\Phi$ is defined on all profiles of $j$ preferences in $\mathbf{E}$, and it satisfies Arrow's three axioms when restricted to allocations of utility value exceeding $k^{51}$

Theorem 9 Limited arbitrage is necessary and sufficient for a resolution of Arrow's paradox on choices of large utility value in the economy $\mathrm{E}$.

Proof. Necessity follows from Proposition 6, since by Arrow's axiom of independence of irrelevant alternatives, the existence of one Condorcet triple of size $k$ suffices to produce Arrow's impossibility theorem on feasible choices of value $k$ in our domain of preferences, see Arrow (1951). Sufficiency is immediate: limited arbitrage eliminates feasible allocation of large utility value by Proposition 1, because it bounds gains from trade. Therefore it resolves Arrow's paradox, because this is automatically resolved in an empty domain of choices.

\subsection{Social choice rules which are continuous, anonymous and respect unanimity}

Consider now the second approach to social choice, Chichilnisky $(1980,1982)$, which seeks continuous anonymous social choice rules which respect unanimity. The link connecting arbitrage with social choices is still very close but it takes a different form. In this case the connection is between the contractibility of the space of preferences, which is necessary and sufficient for the existence of continuous, anonymous rules which respect unanimity (Chichilnisky and Heal (1980)) and limited arbitrage.

Continuity is defined in a standard manner; anonymity means that the social preference does not depend on the order of voting. Respect of unanimity means that if all individuals have identical preferences overall, so does the social preference; it is a very weak version of the Pareto condition. It was shown in Chichilnisky $(1980,1982)$ that, for general spaces of preferences, there exist no social choice rules satisfying these three axioms. Subsequently Chichilnisky and Heal (1983) established that contractibility is exactly what is needed for the existence of social choice rules. It is worth observing that the following result is valid for any topology on the space of preferences $T$. In this sense this result is analogous to a fixed point theorem or to a maximization theorem: whatever the topology, a continuous function from a compact convex space to itself has a fixed point and a continuous function of a compact set has a maximum. All these statements, and the one below, apply independently of the topology chosen: 
Theorem 10 Let $T$ be a connected space of preferences endowed with any topology. ${ }^{52}$ Then $T$ admits a continuous anonymous map $\Phi$ respecting unanimity

$$
\Phi: T^{k} \rightarrow T
$$

for every $k \geq 2$. if and only if $T$ is contractible.

Proof. See Chichilnisky and Heal (1983).

The close relation between contractibility and non-empty intersection (which is limited arbitrage) follows from the following theorem:

Theorem 11 Let $\left\{U_{i}\right\}_{i=1 \ldots I}$ be a family of convex sets in $R^{N}$. The family has a non-empty intersection if and only if every subfamily has a contractible union:

$$
\bigcap_{i=1}^{I} U_{i} \neq \phi \Leftrightarrow \bigcup_{i \in J} U_{i} \text { is contractible } \forall J \subset\{1 \ldots I\} .
$$

Proof. See Chichilnisky (1980 and 1993a).

This theorem holds for general excisive families of sets, including acyclic families and even simple families which consist of sets which need not be convex, acyclic, open or even connected. This theorem was shown to imply the Knaster Kuratowski Marzukiewicz theorem and Brouwer's fixed point theorem (Chichilnisky 1993a), but it is not implied by them. Theorem 11 establishes a close link between contractibility and non-empty intersection and is used to show that limited arbitrage, or equivalently the lack of social diversity, is necessary and sufficient for resource allocation via social choice rules.

Intuitively, a preference is similar to that of trader $h$ when it prefers those allocations which assign $h$ a consumption vector which $u_{h}$ prefers. In mathematical terms this means that the space of preferences similar to those of a subset $J$ of traders in the economy have gradients within the union of the market cones of the traders in $J$. Formally, let the space of choices be $R^{N}$ and define a space of preferences as follows:

Definition 20 Let $P_{J}$ consist of all those preferences which are similar to those of the market economy $\mathrm{E}$, in the sense that their gradients are in the union of the market cones of the traders in $J$, see Chichilnisky (1991, 1991a)

$$
\begin{gathered}
P_{J}=\left\{u: u \text { defines a preference on } R^{N}\right. \text { satisfying Assumption 1, and } \\
\left.\exists J \subset\{1, \ldots, H\}: \forall x \in R^{N}, D u(x) \in \cup_{h \in J} D_{h}\right\} .
\end{gathered}
$$

In the following we assume that for the set $P_{J}$ is connected, for which it suffices that any two traders would wish to trade. ${ }^{53}$

Theorem 12 The economy $E$ satisfies limited arbitrage if and only if for any subset of traders $J \subset\{1,2, \ldots, H\}$ the union of all market cones $\cup_{h \in J} D_{h}$ is contractible. 
Proof. This follows directly from Theorem 11.

Theorem 13 A continuous anonymous social choice rule $\Phi: P_{J}^{k} \rightarrow P_{J}$ which respects unanimity exists which for every $k \geq 2$ and every $J \subset\{1 \ldots . H\}$ if and only if the economy $E$ has limited arbitrage, i.e. if and only if the economy has a competitive equilibrium and a non-empty core.

Proof. See Chichilnisky (1991, 1991a), Theorems 2,7, 10 and 12.

\section{Social Diversity and Limited Arbitrage}

If the economy does not have limited arbitrage, it is called socially diverse:

Definition 21 The economy $\mathbf{E}$ is socially diverse when $\bigcap_{h=1}^{H} D_{h}=\phi$.

This concept is robust under small errors in measurement and is independent of the units of measurement or choice of numeraire. If $E$ is not socially diverse, all economies sufficiently close in endowments and preferences have the same property: the concept is structurally stable. Social diversity admits different "shades"; these can be measured, for example, by the smallest number of market cones which do not intersect:

Definition 22 The economy $\mathrm{E}$ has index of diversity $I(\mathrm{E})=H-K$ if $K+1$ is the smallest number such that $\exists J \subset\{1 \ldots H\}$ with cardinality of $J=K+1$, and $\bigcap_{h \in J} D_{h}=\phi$. The index $I(\mathrm{E})$ ranges between 0 and $H-1$ : the larger the index, the larger the social diversity. The index is smallest when all the market cones intersect: then all social diversity disappears, and the economy has limited arbitrage.

Theorem 14 The index of social diversity is $I(E)$ if and only if $H-I(E)$ is the maximum number of traders for which every subeconomy has a competitive equilibrium, a non-empty core, admits social choice rules which satisfy Arrow's axioms on choices giving large utility values, and admits social choice rules which are continuous, anonymous and respect unanimity on preferences similar to those of the subeconomy.

\section{A topological invariant for the market $\mathrm{E}$}

This section shows that the resource allocation properties of the economy $E$ can be described simply in terms of the properties of a family of cohomology rings denoted $C H(\mathrm{E})$.

A ring is a set $Q$ endowed with two operations, denoted + and $\times$; the operation + must define a group structure for $Q$ (every element has an inverse under + ) and the operation $\times$ defined a semi group structure for $Q$; both operations together satisfy an associative relation. A typical example of a ring is the set of the integers, as well as the rational numbers, both with addition and multiplication. 
The cohomology ring of a space $Y$ contains information about the space's topological structure, namely those properties of the space which remain invariant when the space is deformed as if it was made of rubber. For a formal definition see Spanier (1979). An intuitive explanation is as follows. The cohomology ring consists of maps defined on homology groups. Intuitively a homology group consists of "holes", defined as cycles which do not bound any region in the space $Y$. The homology groups are indexed by dimension. For example the circle $S^{1}$ has the simplest possible "hole": its first homology group measures that. The "torus" $S_{1} \times S_{1}$ has two types of holes: therefore it has a non-zero first homology group as well as a non-zero second homology group. Any convex, or contractible, space has no "holes" so that its cohomology groups are all zero. In addition to the standard group structure of each cohomology group, there is another operation, called a "cup product", which consists, intuitively, of "patching up" elements across cohomology groups. The set of all cohomology groups with these two operations defines the cohomology ring.

The rings $C H(\mathrm{E})$ are the cohomology rings corresponding to subfamilies of market cones $\left\{D_{h}\right\}$ of the economy $E$ define a topological invariant of the economy $E$ in the sense that they are the same for any continuous deformation of the space of commodities on which the economy is defined, i.e. they are preserved under any continuous transformation in the units of measurement of the commodities. They are also preserved under small perturbations of, or measurement errors on, the traders' preferences.

Definition 23 The nerve of a family of subsets $\left\{V_{i}\right\}_{i=1, \ldots, L}$, in $R^{M},{ }^{54}$ denoted

$$
\text { nerve }\left\{V_{i}\right\}_{i=1, \ldots, L}
$$

is a simplicial complex defined as follows: each subfamily of $k+1$ sets in $\left\{V_{i}\right\}_{i=1, \ldots, L}$ with non-empty intersection is a $k$ - simplex of the nerve $\left\{V_{i}\right\}_{i=1, \ldots, L}$.

The topological invariant $C H(\mathrm{E})$ of the economy $\mathrm{E}$ is the family of cohomology rings ${ }^{55}$ of the simplicial complexes defined by all subfamilies $\left\{F_{h}\right\}$ of the family $\left\{D_{h}\right\}_{h=1,2 \ldots H}$, i.e. the cohomology rings of $\left\{\right.$ nerve $\left\{F_{h}\right\}_{h=1 \ldots H}$ where $\left.\left\{F_{h}\right\} \in\left\{D_{h}\right\}\right\}$ :

$$
\left\{H^{*}\left(\text { nerve }\left\{F_{h}\right\}, \forall\left\{F_{h}\right\} \in\left\{D_{h}\right\}\right\}\right.
$$

For the following result I consider continuous deformations of the economy which preserve its convexity and Assumption 1.

Theorem 15 The economy $\mathrm{E}$ with $H$ traders has limited arbitrage, and therefore a competitive equilibrium, a non-empty core and social choice rules if and only if:

$$
\begin{gathered}
C H(\mathrm{E})=0 \\
\text { i.e. } \forall\left\{F_{h}\right\} \in\left\{D_{h}\right\} \mathrm{H}^{*}\left(\text { nerve }\left\{F_{h}\right\}\right)=0
\end{gathered}
$$


Furthermore, the economy $\mathrm{E}$ has social diversity index $I(\mathrm{E})$ if and only if $I(E)=$ $H-K$. where $K$ satisfies the following conditions: (i) for every $\left\{F_{h}\right\} \in\left\{D_{h}\right\}$ of cardinality at most $K$

$$
\mathrm{H}^{*}\left(\text { nerve }\left\{F_{h}\right\}\right)=0 .
$$

and there exists $\left\{J_{h}\right\} \in\left\{D_{h}\right\}$ with cardinality $\left\{J_{h}\right\}=K+1$ and

$$
\mathrm{H}^{*}\left(\text { nerve }\left\{J_{h}\right\}\right) \neq 0 \text {. }
$$

Proof. This follows directly from Chichilnisky (1993c).

\section{Related Literature on Market Equilibrium}

The literature on the existence of a competitive (Walrasian) market equilibrium is about fifty years old, starting with the classic works of Von-Neumann, Nash, Arrow and Debreu and others. This literature has focused on sufficient conditions for existence rather than on necessary and sufficient conditions as studied here. It can be reviewed in two parts: markets without short sales, such as those studied by Arrow and Debreu, and markets with short sales which appear in the literature on financial markets.

\subsection{Related Literature on Equilibrium with Bounds on Short Sales}

Two well known conditions are sufficient for the existence of an equilibrium ${ }^{56}$ when $X=R_{+}^{N}$. They are Arrow's "resource relatedness" condition (Arrow and Hahn, 1971), and McKenzie's "irreducibility" condition (McKenzie 1959, 1961, 1987); both are sufficient but neither is necessary for existence. Both imply limited arbitrage, which is simultaneously necessary and sufficient for the existence of a competitive equilibrium. Resource relatedness and irreducibility ensure existence by requiring that the endowments of any trader are desired, directly or indirectly, by others, so that the treaders' incomes cannot fall to zero. Under these conditions it is easy to check that limited arbitrage is always satisfied, and a competitive equilibrium always exists. Yet traders with zero or minimum income do not by themselves rule out the existence of a competitive equilibrium. Limited arbitrage could be satisfied even when some traders have zero income. This reflects a real situation: some individuals are considered economically worthless, in that they have nothing to offer that others want in a market context. Such a situation could be a competitive equilibrium. Figure 8 provides an example. It seems realistic that markets could lead to such allocations: one observes them all the time in city ghettos. Limited arbitrage does not attempt to rule out individuals with minimum (or zero) income; instead, it seeks to determine if society's evaluation of their worthlessness is shared. Individuals are diverse in the sense of not satisfying limited arbitrage, when someone has minimal (or zero) income, and, in addition, when there is no agreement about the value of those who have minimal income. In such cases there is no competitive equilibrium. 
Another condition which is sufficient but not necessary for existence of a competitive equilibrium is that the indifference surfaces of preferences of positive consumption bundles should be in the interior of the positive orthant, Debreu (1959): this implies that the set of directions along which the utilities increase without bound from initial endowments is the same for all traders. Therefore all individuals agree on choices with large utility values, again a form of similarity of preferences. It is immediate to see that such economies satisfy limited arbitrage.

\subsection{Related Literature on Equilibrium in Markets With Short Sales}

The literature of general equilibrium with short sales has concentrated on sufficient conditions for existence, for example Hart (1974), Kreps (1981) Hammond (1983), Chichilnisky and Heal $(1984,1993)$, and not on the question of conditions which are simultaneously necessary and sufficient for existence as studied here and previously in Chichilnisky (1991, 1992, 1994, 1995) and Chichilnisky and Heal (1992 and 1995). In addition, the literature has neglected economies where the feasible individually rational allocations do not form a compact set. Previous sufficient results for the existence of an equilibrium in economies with short sales rely on the fact that the set of feasible allocations is compact. Theorem 2 above (see also Chichilnisky (1991, 1992, 1994, 1995) and Chichilnisky and Heal (1992 and 1995) is original in that it provides conditions which are necessary and sufficient for existence in economies where feasible and individually rational allocations may be unbounded; in addition these results are novel in that they result apply in economies with or without short sales, and for finitely or infinitely many markets.

In the context of temporary equilibrium models, which are different from Arrow Debreu models because forward markets are missing, Green (1973) established early on interesting necessary and sufficient conditions on "overlapping expectations" for the existence of a temporary equilibrium; similar conditions appear in Grandmont (1982) also in the context of temporary equilibrium and Green's model. Sufficient conditions for existence in economies with short sales, i.e. when $X=R^{N}$, include those of Debreu (1962), which requires the "irreversibility" of the total consumption set $X=\sum_{h=1}^{H} X_{h}$ : this contrasts with limited arbitrage in that it applies to the whole consumption set $X$ rather than to global or market cones, in any case it is only a sufficient condition for existence. Other "no arbitrage" conditions have been used, for example in the finance literature. The connection between the standard notion of no-arbitrage and limited arbitrage, was discussed in Section 2.2. The noarbitrage Condition C of Chichilnisky and Heal $(1984,1993)$ is an antecedent for limited arbitrage: it is a no-arbitrage condition which is sufficient but not necessary in general for the existence of a competitive equilibrium; it requires that along a sequence of feasible allocations where the utility of one trader increases beyond bound, there exists another trader whose utility eventually decreases below this trader's utility at the initial endowment. This result is based on a bounded sets of feasible allocations, a conditions that is not generally satisfied in this paper.

Another condition of no-arbitrage based on recession cones appears in Werner 
(1987) and in Nielsen (1989), who provide sufficient conditions for the existence of an equilibrium in finite dimensions. The results of (1987) and (1989) are posterior and less general than those in those in ${ }^{57}$ Chichilnisky and Heal $(1984,1993)$; they are restricted to finite dimensional economies with short sales and with strictly convex preferences, and are based on bounded sets of feasible allocations. The same noarbitrage condition based on recession cones was used subsequently by Page (1987) for special models of asset prices with strictly convex preferences. which are incomplete markets and exclude also the Arrow Debreu treatment of short sales. The no-arbitrage condition mentioned above is sufficient but not necessary in general economies for the existence of an equilibrium. Under certain conditions which exclude the Arrow Debreu treatment of short sales, and which exclude also the case of preferences which are not strictly convex and which may have different recession cones at different endowments, conditions which are not required here, Werner (1987) provides two conditions, one which is proved to be sufficient for existence of equilibrium and another which is mentioned without formal statement or proof to be necessary for existence. The two conditions involve different cones, and there is no complete proof in Werner (1987) that the two cones, and therefore the two conditions, are the same. However, the two cones in Werner (1987) coincide in very special cases: for example, when recession cones are uniform and equal to directions of strict utility increase and when indifferences contain no halflines, conditions which are not required here. Therefore Werner's comments provide a proof of necessary and sufficient conditions for the special case of economies with short sales, where the recession cones are uniform and coincide with directions of strict utility increase, and when indifferences contain no half-lines, conditions which are explicitly required for example in Page and Wooders certainly do not hold in Werner (1987) nor in this paper. In the general case there is no complete proof in Werner (1987) that his two cones, and therefore his two conditions, are the same; the details are in Section 4 above. No-arbitrage as defined in (Werner 1987) is not defined on initial parameters of the economy: it must be verified in principle at all allocations, thus eliminating cases where limited arbitrage is satisfied (with the same preferences) for some initial endowments and not for others, cases which are included in the analysis of this paper.

\section{Conclusions}

One limitation on social diversity, limited arbitrage, is necessary and sufficient for the existence of a competitive equilibrium, the core and social choice rules in Arrow Debreu economies (Arrow and Debreu, 1954). Social diversity is however more subtle and complex: it comes in many shades. Social diversity is zero when limited arbitrage is satisfied, and it is defined generally in terms of the properties of the cohomology rings $\mathrm{CH}$ of the nerve of a family of cones which are naturally associated with the economy. The cohomology rings of these nerves contain information about which subeconomies have competitive equilibria and a core, and which have social choice rules; the mildest form of social diversity is sufficient for the existence of a supercore, 
which consists of all those allocations which no strict subcoalition has a reason to block.

From these results an implicit prediction emerges about the characteristics of economies which have evolved mechanisms to allocate resources efficiently according to markets, cooperative game solutions, or social choice: they will exhibit only a limited amount of social diversity. Economies which do not succeed in allocating resource efficiently are not likely to be observed in practice, so that existing economies are likely to exhibit limited social diversity.

Other forms of diversity come to mind-for example, the genetic diversity of a population: this is generally believed to be favorable for the species' survival. In the biological context, therefore, diversity appears as a positive feature. This may appear to run counter to what is said here. Not so. Some diversity is desirable in economics as well: as mentioned at the beginning of this paper, without diversity there would be no gains from trade. Indeed without diversity the market would have no reason to exist. The matter is subtle: in the end, it is a question of degrees, of how much diversity is desirable or acceptable.

The tenet of this paper is that the economic organizations which prevail today require a well-defined amount of diversity, and no more, to function properly. One is led to consider the following, somewhat unsettling, question: is it possible that existing forms of economic organization restrict diversity beyond what would be desirable for the survival of our species? Or, more generally: are the forms of social and economic organization which prevail in our society sustainable? 


\section{Notes}

*July 1994, revised July 1995, April 1996 and July 1996. The paper was an invited presentation at the International Economics Association Round Table on Social Choice, Schloss Hernstein, Vienna, Austria, May, 1994. Research support was provided by NSF Grants SBR 92-16028 and DMS 94-08798, and by the Sloan Foundation Project on Information Technology and Productivity. iea8a.tex

+Director, Program on Information and Resources and UNESCO Chair in Mathematics and Economics, Columbia University. Address: 405 Low Library, Columbia University, 116th.and Broadway, New York N.Y. 10025, email: gc9@columbia.edu, fax: 2126780405 . The main results of this paper appeared in "Markets, Arbitrage and Social Choice", paper presented at the October 23, 1991, conference "Columbia Celebrates Arrow's Contributions", Columbia University, New York. Invaluable editorial comments and intellectual input from Kenneth Arrow, Walt Heller, Ted Groves, Morris Hirsch and Wayne Shafer are gratefully acknowledged.

1. The core is an allocation which no subset of players can improve upon within their own endowments.

2. Limited arbitrage was introduced and named in Chichilnisky (1991, 1992, 1993c, 1994, 1994a, 1994b, 1995, 1995a) and Chichilnisky and Heal (1992).

3. No-arbitrage is discussed in Section 2.2 .

4. These results were first established in Chichilnisky (1991, 1992, 1993c, 1994a, 1994b, 1995, 1995a, 1996).

5. This result was first established in Chichilnisky and Heal (1992).

6. This result and its proof were presented at the Econometric Society Meetings in Boston, January 3-5, 1994.

7. A result first established in Chichilnisky (1991,1991a, 1993c, 1994).

8. $C H$ is defined in Section 10.

9. It is possible to use lesser concepts of equilibrium, such as quasiequilibrium and compensated equilibrium, or equilibria where there may be excess supply in the economy. These exist under quite general conditions, but fail to provide Pareto efficient allocations and are therefore less attractive from the point of view of resource allocation, so they are not used here. The relationship between limited arbitrage and quasiequilibrium is explored further in Chichilnisky (1996a).

10. I.e. whether trades are bounded below or not.

11. Contractibility ensures that the preferences of all traders can be continuously deformed into one and is therefore a form of similarity of preferences, see Heal (1983). 
12. This theorem is also valid for non-convex excisive families of sets (Chichilnisky 1980, 1993c), and is shown in to imply Brower's fixed point theorem, the KKM theorem, Caratheodory's theorem and Leray's theorem, but it is not implied by them.

13. In Chichilnisky (1980a, 1993a), a result which has Helly's theorem as a corollary.

14. Arrow and Debreu's formalization of markets assume that the consumption sets of the individuals are bounded below, an assumption motivated by the inability of humans to provide more than a fixed number of hours of labor per day.

15. Chichilnisky and Heal $(1992,1993)$ proved that limited arbitrage is necessary and sufficient for the existence of a competitive equilibrium in economies with infinitely many markets.

16. I work within a standard framework where preferences are convex and uniformly non-satiated, Section 1. These include all standard convex preferences including: linear of partly linear, constant elasticity of substitution (CES), Cobb Douglas, Leontief preferences, strictly convex preferences with indifference surfaces which intersect the coordinate lines or not and which contain half lines or not.

17. Or expectations.

18. As is done in finite dimensions. The Hahn-Banach theorem requires that one of the convex sets being separated has a non-empty interior.

19. See e.g. Chichilnisky (1993a) and more recently Le Van (1996).

20. See also Chichilnisky and Heal (1992).

21. Called the Pareto frontier. The connection between limited arbitrage and the compactness of the Pareto frontier is of central importance for resource allocation. This connection was first pointed out and established in Chichilnisky (1991, 1992, 1994a, 1995) and Chichilnisky and Heal $(1992,1993)$.

22. $R_{+}^{N}=\left\{\left(x_{1}, \ldots, x_{N}\right) \in R^{N}: \forall i, x_{i} \geq 0\right\}$.

23. If $x, y \in R^{N}, x \geq y \Leftrightarrow \forall i x_{i} \geq y_{i} ; x>y \Leftrightarrow x \geq y$ and for some $i, x_{i}>y_{i}$; and $x>>y \Leftrightarrow \forall i, x_{i}>y_{i}$.

24. Namely independent of the utility representations.

25. This means that if $x \in \partial R_{+}^{N}$ and $u(x)>0$, then $D u(x)$ is not orthogonal to $\partial R_{+}^{N}$ at $\lambda x, \forall \lambda \geq 0$. This condition includes strictly convex preferences, Cobb Douglas and CES preferences, many Leontief preferences $u(x, y)=\min (a x, b y)$, preferences which are indifferent to one or more commodities, such as $u(x, y, z)=\sqrt{x+y}$, preferences with indifference surfaces which contain rays of $\partial R_{+}^{N}$ such as $u(x, y, z)=x$, and preferences defined on a neighborhood of the positive orthant or the whole space, and which are increasing along the boundaries, e.g. $u(x, y, z)=x+y+z$. 
26. Smoothness is used to simplify notation only: uniform non satiation requires no smoothness. This is a generalized Liftschitz condition: when preferences admit no smooth utility representation, then one requires $\exists \varepsilon . K>0: K\|x-y\|>|u(y)-u(x)|$ and $\exists \dot{\delta}>0$ : $\sup _{x-y \mid<\delta}|u(y)-u(x)|>\varepsilon\|x-y\|$. for all $x, y \in X$.

27. $N$ is empty when $\forall h, \Omega_{h}>>0$.

28. The global cone $A\left(\rho_{h}, \Omega_{h}\right)$ has points in common with Debreu's (1959) "asymptotic cone" corresponding to the preferred set of $u_{h}$ at the initial endowment $\Omega_{h}$, in that along any of the rays of $A_{h}\left(\Omega_{h}\right)$ utility increases. Under Assumption 1, its closure $\bar{A}\left(\Omega_{h}\right)$, equals the "recession" cone introduced by Rockafeller, but not generally: along the rays in $A\left(\Omega_{h}\right)$ utility increases beyond the utility level of any other vector in the space. This condition need not be satisfied by Debreu's asymptotic cones (Debreu 1959) or by Rockafeller's "recession" cones. For example, for Leontief type preferences the recession cone through the endowment is the closure of the upper contour, which includes the indifference curve itself. By contrast, the global cone $A_{h}\left(\Omega_{h}\right)$ is the interior of the upper contour set. Related concepts appeared in Chichilnisky $(1976,1986)$; otherwise there is no precedent in the literature for global cones. The cones used in the literature on no-arbitrage were Rockafeller's recession cones, until Chichilnisky $(1991,1992)$ and Chichilnisky and Heal (1992).

29. This cone $G_{h}\left(\Omega_{h}\right)$ appears also in Monteiro Page and Wooders (1995) which is a comment on Chichilnisky (1995).

30. The market cone $D_{h}^{+}$is the whole consumption set $X=R^{N+}$ when $S(E)$ has a vector assigning strictly positive income to all individuals. If some trader has zero income, then this trader must have a boundary endowment.

31. A 'boundary ray' $r$ in $R_{+}^{N}$ consists of all the positive multiples of a vector $v \in \partial R_{+}^{N}$ : $r=\left\{w \in R_{+}^{N}: \exists \lambda>0\right.$ s.t. $\left.w=\lambda v\right\}$

32. This includes Cobb-Douglas, constant elasticity of substitution (CES), preferences with indifference surfaces of positive consumption contained in the interior of $R_{+}^{N}$, linear preferences, piecewise linear preferences, most Leontief preferences, preferences with indifference surfaces which intersect the boundary of the positive orthant (Arrow and Hahn (1971)) and smooth utilities defined on a neighborhood of $X$ which are transversal to its boundary $\partial X$.

33. The expression $G(\mathbf{E})<\infty$ holds when $\forall h, \sup _{\{x: x \in X\}} u_{h}(x)=\infty$ as is assumed here; it must be replaced by $G(\mathrm{E})<\sup _{\left\{x: x \in X^{H}: \sum x_{h}=\Omega\right\}}\left(\sum_{h=1}^{H} u_{h}\left(x_{h}\right)-u_{h}\left(\Omega_{h}\right)\right)-k$, for some positive $k$, when $\sup _{\{x: x \in X\}} u_{h}(x)<\infty$.

34. $Z^{c}$ denotes the complement of the set $Z$.

35. A standard example of this phenomenon is in $L_{\infty}=\left\{f: R \rightarrow R: \sup _{x \in R}\|f(x)\|<\right.$ $\infty$ \}. Society's endowment is $\Omega=(1,1, \ldots, 1, \ldots)$, trader one has a preference $u_{1}(x)=\sup \left(x_{i}\right)$, and trader two has a preference $u_{2}(x)=\sum_{i} u\left(x_{i}\right) \lambda^{-i}, 0<\lambda<1$. Then giving one more unit of the ith good to trader two always increases trader two's utility without decreasing that of trader one, and the Pareto frontier cannot be closed, see Chichilnisky and Heal (1993). 
36. Recall that the Pareto frontier is defined as the set of individually rational, feasible and efficient utility allocations, see Section 1.2 .

37. A set $X \subset H$ is bounded below when there exists $y \in H: \forall x \in H, x \geq y$.

38. A topological space $X$ is homeomorphic to another $Y$ when there exists an onto map $f$ : $X \rightarrow Y$ which is continuous and has a continuous inverse.

39. The results on equilibrium in this paper originated from a theorem in Chichilnisky and Heal $(1984,1993)$ a paper which was submitted for publication in 1984, nine years before it appeared in print: these dates are recorded in the printed version. Chichilnisky and Heal (1984, 1993) provided a no-arbitrage condition and proved it is sufficient for the existence of a competitive equilibrium with or without short sales, with infinitely or finitely many markets. See also the following footnote.

40. Chichilnisky and Heal (1984, 1993), Hart (1974) Hammond ((1983) and Werner (1987) among others, have defined various no-arbitrage conditions which they prove, under certain conditions on preferences, to be sufficient for existence of an equilibrium in different models. Except for Chichilnisky and Heal $(1984,1993)$. None of these no-arbitrage conditions is generally necessary for existence. Within econornies with short sales (which exclude Arrow Debreu's markets), and where preferences have no halflines in the indifference surfaces (which exclude "flats"), Werner (1987) remarks (p. 1410, last para.) that another related condition (p. 1410 , line -3 ) is necessary for existence, without however providing a complete proof of the equivalence between the condition which is necessary and that which is sufficient. In general, however, the two conditions in (Werner 1987) are defined on different sets of cones: the sufficient condition is defined on cones $S_{i}$ (p. 1410, line -14) while the necessary condition is defined on other cones, $D_{i}$ (p. 1410, -3). The equivalence between the two cones depends on properties of yet another family of cones $W_{i}$ (see p. 1410, lines 13-4). The definition of $W_{i}$ on page 1408 , line -15 shows that $W_{i}$ is different from the recession cone $R_{i}$, (which are uniform by assumption) and therefore the cone $W_{i}$ need not be uniform even when the recession cones are, as needed in Werner's Proposition 2. His argument for necessity is however complete in a very special case: when preferences have uniform recession cones, the recession cones coincide with directions of strict utility increase and indifferences have no half lines. In general, however, even for the special case of economies with short sales and with strictly convex preferences, Chichilnisky (1991, 1992, 1994 and 1995) and the results presented here appear to provide the first complete proof of a condition (limited arbitrage) which is simultaneously necessary and sufficient for the existence of a competitive equilibrium.

41. The no-arbitrage conditions in Chichilnisky and Heal (1984, 1993), and Werner (1987) do not provide necessary and sufficient conditions for all the economies considered in this paper: all prior results (except for those in Chichilnisky 1991, 1992, 1994,1995) depend crucially on the fact that the set of feasible allocations is compact. By contrast, the boundedness of feasible allocations is neither required, nor it is generally satisfied, in the economies considered in this paper, because although the feasible allocations may be unbounded, there exists a bounded set of allocations which reach all possible feasible utility levels. 
42. A pseudoequilibrium, also called quasiequilibrium, is an allocation which clears the market and a price vector at which traders minimize cost within the utility levels achieved at their respective allocations. The connection between limited arbitrage and quasiequilibrium is studied in Chichilnisky (1996a). For the proof of existence of a quasiequilibrium cf. Negishi (1960), who studied the case where the economy has no short sales. For cases where short sales are allowed, and therefore feasible allocations may be unbounded, a method similar to Negishi's can be used, see e.g. Chichilnisky and Heal (1984, 1993) and Chichilnisky (1991, 1992, 1994,1995). With strictly convex preferences, limited arbitrage implies that feasible allocations form a bounded set; otherwise, when indifferences have "flats", the set of feasible allocations may be unbounded. However in this latter case there exists a bounded set of feasible allocations which achieves all feasible utility levels, and this suffices for a Negishi-type proof of existence to go through.

43. Also known in subsequent work as "properness", see Chichilnisky (1993) and Le Van (1996).

44. I proved this result in the finite dimensional case while at Stanford University in the Spring and Summer of 1993, stimulated by conversations with Curtis Eaves, and presented this result and its proof at the January 3-5, 1994 Meetings of the Econometric Society in Boston.

45. See also Chichilnisky $(1994,1996)$.

46. In the economy $E$ the traders' preferences are defined over private consumption $u_{i}: R^{N} \rightarrow$ $R$, but they define automatically preferences over allocations in $R^{N \times H}: u_{i}\left(x_{1} \ldots x_{H}\right) \geq$ $u_{i}\left(y_{1} \ldots y_{H}\right) \Leftrightarrow u_{i}\left(x_{i}\right) \geq u_{i}\left(y_{i}\right)$.

47. See also Chichilnisky (1982).

48. A space $X$ is contractible when there exists a continuous map $f: X \times[0,1] \rightarrow X$ and $x_{o} \in X$ such that $\forall x, f(x, 0)=x$ and $f(x, 1)=x_{0}$.

49. The concept of "large utility values" is purely ordinal; it is defined relative to the maximum utility value achieved by a utility representation.

50. If indifferences are bounded below, nothing is required of the sets of gradients. These conditions can be removed, but at the cost of more notation.

51. Recall that we have assumed, without loss, that $\sup _{x \in X} u_{h}(x)=\infty$. Otherwise the same statement holds by replacing " $>k$ " by " $>\sup _{x \in X} u_{h}(x)-k$."

52. $T$ could be the space of linear preferences on $R^{N}$ or the space of strictly convex preferences on $R^{N}$, or the space of all smooth preferences. $T$ could be endowed with the closed convergence topology, or the smooth topology, or the order topology, etc. $T$ must satisfy a minimal regularity condition, for example to be locally convex (every point has a convex neighborhood) or, more generally, to be a parafinite $\mathrm{CW}$ complex. This is a very general specification, and includes all the spaces used routinely in economics, finite or infinite dimensional, such as all euclidean spaces, Banach and Hilbert spaces, manifolds, all piece-wise linear spaces, polyhedrons, simplicial complexes, or finite or infinite dimensional CW spaces. 
53. Since we apply Theorem 11, we require that the space of preferences $P_{J}$ be connected. In a market economy, this requires that every two traders have a reason to trade, but says nothing about sets of three or more traders, nor does it imply limited arbitrage.

j4. $\forall i . V_{i} \subset R^{M}$.

55. With integer coefficients.

56. Not all Arrow-Debreu exchange economies have a competitive equilibrium, even when all individual preferences are smooth, concave and increasing, and the consumption sets are positive orthants, $X=R_{+}^{N}$, see for example Arrow and Hahn (1971), Chapter 4, p. 80 .

57. The results on existence of an equilibrium in Chichilnisky and Heal $(1984,1993)$ (which are valid in finite or infinite dimensional economies) contain as a special case the results on existence of equilibrium in Werner (1987). The no-arbitrage Condition $\mathrm{C}$ introduced by Chichilnisky and Heal $(1984,1993)$ is weaker that the no-arbitrage condition defined by Werner (1987). As recorded in its printed version, Chichilnisky and Heal (1993) was submitted for publication in February 1984. As recorded in its printed version, Werner's paper (1984) was subrnitted for publication subsequently, in July 1985

\section{References}

Arrow, K.(1951) Social Choice and Individual Values, (New York: Cowles Foundation Monograph, John Wiley and Sons).

Arrow, K., and F. Hahn (1971) General Competitive Analysis, (San Francisco and New York: North Holland), 1986.

Arrow, K. and G. Debreu (1954) 'Existence of an Equilibrium for a Competitive Economy,' Econometrica Vol. 22, pp. 264-90.

Baryshnikov, Y. (1993) 'Unifying Impossibility Theorems: A Topological Approach,' Advances in Applied Mathematics, Vol. 14, pp. 404-415.

Black, D. (1948) 'The Decisions of a Committee using a Simple Majority,' Econometrica, Vol. 16, pp. 245-61.

Chichilnisky, G. (1976) 'Manifolds of Preferences and Equilibria,' Ph.D. Dissertation, Department of Economics, University of California, Berkeley, USA, published as Discussion Paper of the Project on Efficiency of Decision Making in Economic Systems,

Harvard University, Cambridge, USA.

Chichilnisky, G. (1980) 'Social Choice and the Topology of Spaces of Preferences,' Advances in Mathematics, Vol. 37, No. 2, pp. 165-176.

Chichilnisky, G. (1980a) 'Intersecting Families of Sets' Working Paper, University of Essex, U.K.

Chichilnisky, G. (1982) 'Social Aggregation Rules and Continuity,' Quarterly Journal of Economics, Vol. XCVII, May 1982, pp. 337-352.

Chichilnisky, G. (1986) 'Topological Complexity of Manifolds of Preferences,' (W. Hildenbrand and A. Mas-Colell eds.) Essays in Honor of Gerard Debreu (New York: North-Holland) Chapter 8, pp. 131-142. 
Chichilnisky, G. (1991) 'Markets, Arbitrage and Social Choice,' paper presented at the conference 'Columbia Celebrates Arrow's Contributions' Columbia University, New York, October 27, 1991, Working Paper No. 586, Columbia University, December 1992, and CORE Discussion Paper No. 9342, CORE, Universite Catolique de Louvain, Louvain la Neuve, Belgium, 1993.

Chichilnisky, G. (1991a) 'Market Arbitrage, Social Choice and the Core,' Social Choice and Welfare. 1996 (forthcoming).

Chichilnisky,G. (1992) 'Limited Arbitrage is Necessary and Sufficient for the Existence of a Competitive Equilibrium,' Working Paper No. 650, Columbia University Department of Economics, December 1992.

Chichilnisky, G. (1993) 'On Strategic Control' Quarterly Journal of Economics, Vol. 108, February 1993, pp. 285-290.

Chichilnisky, G.(1993a) 'The Cone Condition, Properness and Extremely Desirable Commodities,' Economic Theory, Vol. 3, pp. 177-82.

Chichilnisky, G. (1993b) 'Topology and Economics: the Contribution of Stephen Smale,' (M. Hirsch, J. Marsden, and M. Shub. eds.) From Topology to Computation, Proceedings of the Smalefest (New York-Heidelberg:Springer Verlag), pp. 147-161.

Chichilnisky, G. (1993c) 'Intersecting Families of Sets and the Topology of Cones in Economics,' Bulletin of the American Mathematical Society, October 1993, Vol. 29, No. 2, pp. 189-207, first circulated as University of Essex Working Paper, 1980.

Chichilnisky, G. (1996) 'Limited Arbitrage is Necessary and Sufficient for the Nonemptiness of the Core', Economic Letters, August-September 1996 (in press), Presented and distributed at the Yearly Meetings of the American Economic Association, Boston, January 3-5, 1994, previously published as 'Limited Arbitrage is Necessary and Sufficient for the Existence of a Competitive Equilibrium and the Core and it Limits Voting Cycles' Economic Letters, (1994a), Vol. 46, December 1994, pp. 321-331 in an issue which was subsequently reprinted by the editor without this paper.

Chichilnisky, G. (1994b) 'Limited Arbitrage, Gains from Trade and Social Diversity: A Unified Perspective on Resource Allocation,' Working Paper, Columbia University, American Economic Review, Vol. 84, No. 2, May 1994, pp. 427-434.

Chichilnisky, G. (1995) 'Limited Arbitrage is Necessary and Sufficient for the Existence of a Competitive Equilibrium With or Without Short Sales,' Working Paper, Columbia University, March 1991, Economic Theory, Vol. 5, No. 1, pp. 79-108, January 1995.

Chichilnisky, G. (1995a) 'A Unified Perspective on Resource Allocation: Limited Arbitrage is Necessary and Sufficient for the Existence of a Competitive Equilibrium, the Core and Social Choice,' CORE Discussion Paper No. 9527, Universite Catolique de Louvain, Louvain-la-Neuve, Belgium, April 1995.

Chichilnisky, G. (1996a) 'A Topological Invariant for Competitive Markets' Working Paper, Columbia University, May 1996, Journal of Mathematical Economics (forthcoming).

Chichilnisky (1996) 'Limited Arbitrage is Necessary and Sufficient for the Exis- 
tence of a Competitive Equilibrium' Journal of Mathematical Economics, forthcoming.

Chichilnisky, G. (1996) 'Topology and Invertible Maps' Working Paper Columbia University, June 1996

Chichilnisky, G. 91996) 'Limited Arbitrage and Uniqueness of Equilibrium in Strictly Regular Economies' Working Paper, Columbia University July 1996.

Chichilnisky, G. and Kalman P. J. (1980) 'Application of Functional Analysis to the Efficient Allocation of Economic Resources,' Journal of Optimization Theory and Applications, Vol. 30, No. 1, pp. 19-32.

Chichilnisky, G. and Heal, G. M. (1983) 'Necessary and Sufficient Conditions for a Resolution of the Social Choice Paradox,' Journal of Economic Theory, Vol. 31, No. 1, pp. 68-87.

Chichilnisky, G. and Heal G.M. (1992) 'Arbitrage and Equilibrium in Sobolev Spaces,' First Boston Working Paper, Columbia University, revised in February 1995 under the title: 'Equilibrium and the Core with Finitely or Infinitely Many Markets: A Unified Approach,; to appear in Economic Theory.

Chichilnisky, G. and G. M. Heal (1993) 'Existence of a Competitive Equilibrium in Sobolev Spaces without Bounds on Short Sales,' IMA Preprint series No. 79, Institute for Mathematics and its Applications, University of Minnesota, Minneapolis, Minnesota, June 1984. Submitted for publication in February 28, 1984, Journal of Economic Theory Vol. 59, No. 2, pp. 364-384.

Debreu, G. (1959) The Theory of Value (New York: Cowles Foundation Monograph, John Wiley).

Debreu, G. (1962) 'New Concepts and Techniques in Equilibrium Analysis,'

International Economic Review, Vol 3, pp. 257-73.

Grandmont, J. M. (1982) 'Temporary Equilibrium' ( Arrow K. and Intriligator M. eds.) Handbook of Mathematical Economics (New York: North Holland).

Green, J. (1973) 'Temporary Equilibrium in a Sequential Trading Model with Spot and Futures Transactions,' Econometrica, Vol. 41, No. 6, pp. $1103-23$.

Hammond, P. (1983) 'Overlapping Expectations and Hart's Conditions for Equilibrium in a Securities Market' Journal of Economic Theory, Vol. 31, pp. 170-75.

Hart, O. (1974) 'Existence of Equilibrium in a Securities Model' Journal of Economic Theory, Vol. 9, pp. 293-311.

Heal, G. M. (1983) 'Contractibility and Public Decision Making”,' (PattanaikP. and Salles S. eds) Social Choice and Welfare, (New York: North Holland) Chapter 7.

Kreps, D. (1981) 'Arbitrage and Equilibrium in Economies with Infinitely Many Commodities,' Journal of Mathematical Economics, Vol. 8, pp. 15-35.

Le Van, A. (1996) 'Complete Characterization of Yannelis-Zame and ChichilniskyKalman-Mas Colell properness conditions on preferences for separable concave functions defined on $L_{+}^{p}$ and $L^{p}$.' Economic Theory, Vol. 8, No. 1, June 1996, pp. $155-166$.

McKenzie, L. (1959) 'On the existence of a general equilibrium for competitive markets,' Econometrica, Vol. 27, pp. 54-71. 
McKenzie, L.(1987) 'General Equilibrium', General Equilibrium, The New Palgrave (Eatwell J., Milgate, M. and Newman P. eds.) (New York:Norton).

McKenzie, L. (1961) 'On the Existence of General Equilibrium: Some Corrections, Econometrica, Vol. 29, pp. 247-248.

Koutsougeras, L. (1993) 'The core in two-stage games' Working Paper, University of Mlinois. Lrbana. Mlinois.

Negishi, T. (1960) 'Welfare Economics and the Existence of an Equilibrium for a Competitive Economy,' Metroeconomica Vol. 2, pp. 92-97.

Nielsen, L. (1989) 'Asset Market Equilibrium with Short Selling' Review of Economic Studies, Vol. 56., No. 187 , pp. 467-473.

Page, F. (1987) 'Notes and Comments to the Editor: On Equilibrium in Hart's Securities Exchange Model' Journal of Economic Theory, Vol. 41, pp. 392-404.

Spanier, E. (1979) Algebraic Topology, (NewYork: McGraw Hill).

Werner, J. (1987) 'Arbitrage and the Existence of Competitive Equilibrium,' Econometrica Vol. 55, No. 6, pp. 1403-1418. 


\title{
1995-1996 Discussion Paper Series
}

\author{
Department of Economics \\ Columbia University \\ 1022 International Affairs Bldg. \\ 420 West 118th Street \\ New York, N.Y., 10027
}

The following papers are published in the 1995-96 Columbia University Discussion Paper series which runs from early November to October 31 of the following year (Academic Year).

Domestic orders for discussion papers are available for purchase at the cost of $\$ 8.00$ (U.S.) Per paper and $\$ 140.00$ (US) for the series.

Eoreign orders cost $\$ 10.00$ (US) per paper and $\$ 185.00$ for the series.

To order discussion papers, please write to the Discussion Paper Coordinator at the above address along with a check for the appropriate amount, made payable to Department of Economics, Columbia University. Please be sure to include the series number of the requested paper when you place an order. 


\section{5-96 Discussion Paper Series}

9596-01 Protectionist Response to Import Competition in Declining Industries Reconsidered

9596-02 New Estimates on Climate Demand: Evidence from Location Choice

9596-03 Enforcement by Hearing

9596-04 Preferential Trading Areas and Multilateralism: Strangers, Friends or Foes?

9596-05 Simplification, Progression and a Level Playing Field

9596-06 The Burden of Proof in Civil Litigation

9596-07 Market Structure and the Timing of Technology Adoption

9596-08 The Emergence of the World Economy

9596-09 The Global Age: From a Skeptical South to a Fearful North

9596-10 A Conformity Test for Cointegration

9596-11 Identification and Kullback Information in the GLSEM

9596-12 Informational Leverage and the Endogenous Timing of Product Introductions

9596-13 Changes in Wage Inequality

9596-14 The Design of Monte Carlo Experiments for VAR Models

9596-15 A Toplogical Invariant for Competitive Markets

9596-16 Topology and Invertible Maps

9596-17 Smooth Infinite Economies by: J. Choi

by: M Cragg

M Kahn

by: C. Sanchirico

by: J. Bhagwati

A. Panagariya

by: W Vickrey

by: C. Sanchirico

by: J. Choi

M Thum

by: R. Findlay

by: J. Bhagwati

by: P. Dhrymes

by: P. Dhrymes

by: J. Choi

by: J. Mincer

by: P. Dhrymes

by: G. Chichilnisky

by: G. Chichilnisky

by: G. Chichilnisky 


\section{5-96 Discussion Paper Series}

9596-18 Measuring Neighborhood Investments: Urban Quality of Life Expenditures by Race

9596-19 The Silver Lining of Industrial Decline: Rust Belt Manufacturing's Impact on Particulates

$9596-20$

$9596-21$

$9596-22$

$9596-23$

9596-24

9596-25

$9596-26$

Limited Arbitrage and Uniqueness of Market Equilibrium in Strictly Regular Economies
A Probabilistic Model of Learning in Games

Minimal inclusive Sets in Special Classes of Games

'Globalization' and Vertical Structure

Corruption, Black Markets and the Fiscal Problem in LDC's: Some Recent Findings

Black Markets and Optimal Evadable Taxation

9596-27 What Drives Deforestation in the Brazilian Amazon? Evidence from Satellite and Socioeconomic Data

9596-28 A Unified Perspective on Resource Allocation: Limited Arbitage is Necessary and Sufficient for the Existence of a Competitive Equilibrium, the Core and Social Choice

9596-29 Smooth Infinite Economies

9596-30 Market Arbitage, Social Choice and the Core

9596-31 The Structural Barrier to Transition: A Note on Input-Output Tables of Centrally Planned Economies

9596-32 When and How to Vote: Resolving Non-contractible Uncertainty Efficiently

9596-33 The Form of U.S. In-Kind Assistance

9596-34 Mixing Government with Voluntaryism by: D. Dipasquale

M Kahn

by: M Kahn

by: M Kahn

by: G. Chichilnisky

by: C. Sanchirico

by: C. Sanichirico

by: J. McLaren

by: J. McLaren

by: J. McLaren

by: A. Pfaff

by: G. Chichilnisky

by: G. Chichilnisky

Y. Zhou

by: G. Chichilnisky

by: R. Ericson

by: B. O'Flaherty

by: B. O'Flaherty

by: K. Lancaster 